\title{
ON PISOT'S $d$-TH ROOT CONJECTURE FOR FUNCTION FIELDS AND RELATED GCD ESTIMATES
}

\author{
JI GUO, CHIA-LIANG SUN, AND JULIE TZU-YUEH WANG
}

\begin{abstract}
We propose a function-field analog of Pisot's $d$-th root conjecture on linear recurrences, and prove it under some "non-triviality" assumption. Besides a recent result of Pasten-Wang on Büchi's $d$-th power problem, our main tool, which is also developed in this paper, is a function-field analog of an GCD estimate in a recent work of Levin and Levin-Wang. As an easy corollary of such GCD estimate, we also obtain an asymptotic result.
\end{abstract}

\section{INTRODUCTION}

For a field $k$, we denote by $\bar{k}$ its algebraic closure. Let $R(X)=\sum_{n=0}^{\infty} b(n) X^{n}$ represent a rational function in $\mathbb{Q}(X)$ and suppose that $b(n)$ is a $d$-th power in $\mathbb{Q}$ for all large $n \in \mathbb{N}$. Pisot's $d$-th root conjecture states that one can choose a $d$-th root $a(n)$ of $b(n)$ such that $\tilde{R}(X):=\sum a(n) X^{n}$ is again a rational function in $\overline{\mathbb{Q}}(X)$. The sequence $\{b(n)\}$ coming from the rational function $R(X)$ is a linear recurrence sequence, which can be written as an exponential polynomial, which we now define. An exponential polynomial over a field $k$ is a sequence $b: \mathbb{N} \rightarrow k$ of the form

$$
n \mapsto \sum_{i \in I} B_{i}(n) \beta_{i}^{n},
$$

where $I$ is a (finite) set of indices, each $\beta_{i} \in k^{*}$ is nonzero and each $B_{i} \in k[T]$ is a single-variate polynomial. When it can be arranged so that each $B_{i}$ is constant, we say that $b$ is simple. For any exponential polynomial $b: \mathbb{N} \rightarrow k$ and any natural number $d$, it is clear that $b^{d}: \mathbb{N} \rightarrow k$, defined by $n \mapsto b(n)^{d}$, is still an exponential polynomial. The following result of Zannier [15] essentially proves its converse, which is a generalization of Pisot's $d$-th root conjecture stated earlier. We also refer to [15] for a survey on related works.

Theorem 1 (15]). Let $b$ be an exponential polynomial over a number field $k$, and $d \geq 2$ be an integer. Suppose that $b(n)$ is the $d$-th power of some element in $k$ for all but finitely many $n$. Then there exists an exponential polynomial a over $\bar{k}$ such that $a(n)^{d}=b(n)$ for all $n$.

The main purpose of this paper is to investigate a function-field analog of Theorem 1 .

Let $C$ be a smooth projective algebraic curve of genus $\mathfrak{g}$ defined over an algebraically closed field $\mathbf{k}$ of characteristic zero. Let $K:=\mathbf{k}(C)$ be its function field. We will always denote by $\mathfrak{p}$ a point in $C(\mathbf{k})$, by $S$ a finite subset of $C(\mathbf{k})$. Since $K$ contains the algebraically-closed field $\mathbf{k}$, note that for any pair of exponential

2020 Mathematics Subject Classification: Primary 11D61; Secondary 14H05 and 11B37.

The second named author was supported in part by Taiwan's MoST grant 107-2115-M-001-013-MY2, and the third named author was supported in part by Taiwan's MoST grant 108-2115-M-001-001-MY2. 
polynomials $a: \mathbb{N} \rightarrow K$ and $c: \mathbb{N} \rightarrow \mathbf{k}$ we have that $c a^{d}: \mathbb{N} \rightarrow K$, defined by $n \mapsto c(n) a(n)^{d}$, is still an exponential polynomial whose $n$-th term is the $d$-th power of some element in $K$ for all $n \in \mathbb{N}$. A plausible statement obtained from Theorem 1 by replacing the number field $k$ by our function field $K$ must therefore have its conclusion modified to the existence of exponential polynomials $a, c$, respectively over $\bar{K}$ and over $\mathbf{k}$, such that $c(n) a(n)^{d}=b(n)$ for all $n$. Our result in this direction is as follows.

Theorem 2. Let $b(n)=\sum_{i=1}^{\ell} B_{i}(n) \beta_{i}^{n}$ be an exponential polynomial over $K$, i.e. $B_{i} \in K[T]$ and $\beta_{i} \in K^{*}$. Let $\Gamma$ be the multiplicative subgroup of $K^{*}$ generated by $\beta_{1}, \ldots, \beta_{\ell}$. Assume that $\Gamma \cap \mathbf{k}^{*}=\{1\}$. If $b(n)$ is a d-th power in $K$ for infinitely many $n \in \mathbb{N}$, then there exists an exponential polynomial $a(m)=\sum_{i=1}^{r} A_{i}(m) \alpha_{i}^{m}$, $A_{i} \in \bar{K}[T], \alpha_{i} \in \bar{K}^{*}$, and a polynomial $R \in \mathbf{k}[T]$ such that $b(m)=R(m) a(m)^{d}$ for all $m \in \mathbb{N}$.

Remark 3. The assumption that $b(n)$ is a $d$-th power in $K$ for infinitely any $n \in \mathbb{N}$ is weaker than the one in Theorem 1. We refer to [4 for a result over number field under both this weaker assumption and the existence of a dominant $\beta_{i}$, i.e. a unique $\beta_{i}$ of maximal or minimal absolute value.

Remark 4. In the notation of Theorem[2 it is standard to notice that $b$ consists of the $q$ disjoint subsequences $b_{j}$, defined by $n \mapsto b(j+q n)$, where $j \in\{0, \ldots, q-1\}$ and $q$ is the order of the torsion subgroup of $\Gamma$; moreover, each $b_{j}$ is an exponential polynomial whose associated $\Gamma_{j}$ is torsion-free. With this observation, we may generalize Theorem 2 so that the assumption $\Gamma \cap \mathbf{k}^{*}=\{1\}$ is relaxed to that $\Gamma \cap \mathbf{k}^{*}$ is finite and the conclusion only holds for some $b_{j}$ rather than $b$.

Remark 5. In the case where $b$ is simple, i.e., each $B_{i}$ is constant, we can relax the hypothesis on $\Gamma$ in Theorem 2 so that the case where $\Gamma \cap \mathbf{k}^{*}$ is infinitely cyclic generated by $\gamma$ can be also treated. In this new case, modifying slightly our proof of Theorem 2, we can conclude that $b(m)=c(m) a(m)^{d}$ for all $m \in \mathbb{N}$, where $c$ is a simple exponential polynomial over $\mathbf{k}$ given by $m \mapsto \sum_{i=1}^{r} c_{i} \gamma^{e_{i} m}$ for some $c_{i} \in \mathbf{k}$ and $e_{i} \in \mathbb{Z}$. It seems difficult to further relax the hypothesis on $\Gamma$.

Our proof of Theorem 2 contains two major ingredients, both rely on the special features of function fields of characteristic zero. One of the ingredients is the result (restated as Theorem 23 in Section 4) by Pasten and Wang 12 motivated by Büchi's $d$-th power problem, which has a similar flavor as Pisot's $d$-th root conjecture but arising from different purposes. While working on an undecidability problem related to Hilbert's tenth problem in the 1970s, Büchi formulated a related arithmetic problem, which can be stated in more generality as follows: Let $k$ be a number field. Does there exist an integer $M$ such that the only monic polynomials $G \in k[T]$ of degree $d$ satisfying that $G(1), \ldots, G(M)$ are $d$-th powers in $k$, are precisely those of the form $G(X)=(X+c)^{d}$ for some $c \in k$. This problem remains unsolved, while its analogs have been investigated intensively in the recent years. In particular, the analog over function fields of characteristic zero was solved completely, even with an explicit bound on $M$; see [2] and [1]. We refer to [12] for a survey of relevant works. The other ingredient, which is also developed in this paper, is the function-field analog of the recent work of Levin [7] for number fields and Levin-Wang [8] for meromorphic functions on GCD 
estimates of two multivariable polynomials over function fields evaluated at arguments which are $S$-units. We will use the estimates through the following result, which is of independent interest.

Theorem 6. Let $d \geq 2$ be an integer and $F \in K\left[x_{1}, \ldots, x_{n}\right]$. Assume that $F$ can not be expressed as $a \mathbf{x}^{\mathbf{i}} G^{d}$ for any $a \in K^{*}$, any monomial $\mathbf{x}^{\mathbf{i}} \in K\left[x_{1}, \ldots, x_{n}\right]$, and any $G \in K\left[x_{1}, \ldots, x_{n}\right]$. Then we have the following conclusion: For any $u_{1}, \ldots, u_{n} \in \mathcal{O}_{S}^{*}$, there exists positive integer $m$ and rationals $c_{1}$ and $c_{2}$ all depending only on $\left(d, n, \operatorname{deg} F, \max _{1 \leq j \leq n} h\left(u_{j}\right)\right)$, such that if $F\left(u_{1}^{\ell}, \ldots, u_{n}^{\ell}\right)$ is a d-th power in $K$ with some $\ell \geq c_{1} \tilde{h}(F)+c_{2} \max \{1,2 \mathfrak{g}-2+|S|\}$, then $u_{1}^{m_{1}} \cdots u_{n}^{m_{n}} \in \mathbf{k}$ for some $\left(m_{1}, \ldots, m_{n}\right) \in \mathbb{Z}^{n} \backslash\{(0, \ldots, 0)\}$ with $\sum\left|m_{i}\right| \leq 2 m$.

Here $\tilde{h}(F)$ is the relevant height of $F$ to be defined in the next section.

Remark 7. We cannot drop $a$ in the assumption of Theorem [6] For example, if $a=u_{1} \in \mathcal{O}_{S}^{*}$ and $F\left(x_{1}\right):=a x_{1}$, we always have that $F\left(u_{1}^{d \ell-1}\right)$ is a $d$-th power in $K$ for all $\ell \in \mathbb{N}$.

The assumption in Theorem 2 that $\Gamma \cap \mathbf{k}^{*}$ is trivial implies that every minimal set of generators of $\Gamma$ is multiplicatively independent modulo $\mathbf{k}$. This suggests how Theorem 6 plays a role in our proof of Theorem 2.

We briefly describe the core idea connecting GCD estimates and our proof of Theorem [6, as introduced by Corvaja-Zannier [5]. After some reduction, we only need to treat the case where $F$ is $d$-th power free. Given a tuple $\left(u_{1}, \ldots, u_{n}, y\right) \in\left(\mathcal{O}_{S}^{*}\right)^{n} \times K$ satisfies that $y^{d}=F\left(u_{1}, \ldots, u_{n}\right)$, we will construct a polynomial $G \in K\left[x_{1}, \ldots, x_{n}\right]$ with controllable height, depending on $F$ and the $\frac{u_{i}^{\prime}}{u_{i}}$, such that $\left(y^{d}\right)^{\prime}=G\left(u_{1}, \ldots, u_{n}\right)$, where ' denotes a global derivation on $K$. For example, if $F:=x_{1}^{2}+\cdots+x_{n}^{2}$, then our construction will yield $G:=2 \frac{u_{1}^{\prime}}{u_{1}} x_{1}^{2}+\ldots+2 \frac{u_{n}^{\prime}}{u_{n}} x_{n}^{2}$. As $d \geq 2$, the number of common zeros of $y^{d}$ and $\left(y^{d}\right)^{\prime}$ is essentially larger than the number of zeros of $y^{d-1}$. On the other hand, we expect the number of common zeros of $F\left(u_{1}, \ldots, u_{n}\right)$ and $G\left(u_{1}, \ldots, u_{n}\right)$ to be essentially smaller than the number of zeros of $y^{d-1}$ unless something special happens. To formalize this idea, we prove the following result on GCD estimates, where all notation involved are defined in Section 2 ,

Theorem 8. Let $S \subset C$ be a finite set of points. Let $F, G \in K\left[x_{1}, \ldots, x_{n}\right]$ be a coprime pair of nonconstant polynomials. For any $\epsilon>0$, there exist an integer $m$, positive reals $c_{i}, 0 \leq i \leq 4$, all depending only on $\epsilon$, such that for all $n$-tuple $\left(g_{1}, \ldots, g_{n}\right) \in\left(\mathcal{O}_{S}^{*}\right)^{n}$ with

$$
\max _{1 \leq i \leq n} h\left(g_{i}\right) \geq c_{1}(\tilde{h}(F)+\tilde{h}(G))+c_{2} \max \{0,2 \mathfrak{g}-2+|S|\}
$$

we have that either

$$
h\left(g_{1}^{m_{1}} \cdots g_{n}^{m_{n}}\right) \leq c_{3}(\tilde{h}(F)+\tilde{h}(G))+c_{4} \max \{0,2 \mathfrak{g}-2+|S|\}
$$

holds for some integers $m_{1}, \ldots, m_{n}$, not all zeros with $\sum\left|m_{i}\right| \leq 2 m$, or the following two statements hold.

(a) $N_{S, g c d}\left(F\left(g_{1}, \ldots, g_{n}\right), G\left(g_{1}, \ldots, g_{n}\right)\right) \leq \epsilon \max _{1 \leq i \leq n} h\left(g_{i}\right)$; 
(b) $h_{\text {gcd }}\left(F\left(g_{1}, \ldots, g_{n}\right), G\left(g_{1}, \ldots, g_{n}\right)\right) \leq \epsilon \max _{1 \leq i \leq n} h\left(g_{i}\right)$ if we further assume that not both of $F$ and $G$ vanish at $(0, \ldots, 0)$.

Remark 9. In Theorem 8 , all the quantities claimed to exist can be given effectively. Moreover, the explicit bounds on heights are important in our application. As said earlier, if we are given $F=x_{1}^{2}+\cdots+x_{n}^{2}$ in Theorem [6, then in the main step of the proof, we construct $G_{\mathbf{u}}:=2 \frac{u_{1}^{\prime}}{u_{1}} x_{1}^{2}+\ldots+2 \frac{u_{n}^{\prime}}{u_{n}} x_{n}^{2}$ for each tuple $\mathbf{u}:=\left(u_{1}, \ldots, u_{n}\right) \in\left(\mathcal{O}_{S}^{*}\right)^{n}$ and apply Theorem 8 to estimate the GCD of $F(\mathbf{u})$ and $G_{\mathbf{u}}(\mathbf{u})$. The main point which makes the proof of Theorem $\left[6\right.$ works is that $\tilde{h}\left(G_{\mathbf{u}}\right)$ can be explicitly bounded independent of these $\mathbf{u}$. (See Proposition [17)

It is more desirable to obtain GCD estimates, such as Statement (a) and (b) in Theorem 8, under the assumption that $g_{1}, \ldots, g_{n}$ are multiplicatively independent modulo $\mathbf{k}$. As a result in this direction, we can actually replace the right hand side of (1.2) by 0 in the case where $n=2$ and the coefficients of $F$ and $G$ are in $\mathbf{k}$. We include a complete statement below. Although this result can be deduced from [5, Corollary $2.3]$, we will derive it from our proof of Theorem 8

Theorem 10. Let $F, G \in \mathbf{k}\left[x_{1}, x_{2}\right]$ be nonconstant coprime polynomials. For any $\epsilon>0$, there exist an integer $m$, constant $c$, both depending only on $\epsilon$, such that for all pairs $\left(g_{1}, g_{2}\right) \in\left(\mathcal{O}_{S}^{*}\right)^{2}$ with $\max \left\{h\left(g_{1}\right), h\left(g_{2}\right)\right\} \geq$ $c \max \{1,2 \mathfrak{g}-2+|S|\}$, either we have that $g_{1}^{m_{1}} g_{2}^{m_{2}} \in \mathbf{k}$ holds for some integers $m_{1}, m_{2}$, not all zeros with $\left|m_{1}\right|+\left|m_{2}\right| \leq 2 m$, or the following two statements hold

(a) $N_{S, \text { gcd }}\left(F\left(g_{1}, g_{2}\right), G\left(g_{1}, g_{2}\right)\right) \leq \epsilon \max \left\{h\left(g_{1}\right), h\left(g_{2}\right)\right\}$;

(b) $h_{\text {gcd }}\left(F\left(g_{1}, g_{2}\right), G\left(g_{1}, g_{2}\right)\right) \leq \epsilon \max \left\{h\left(g_{1}\right), h\left(g_{2}\right)\right\}$, if we further assume that not both of $F$ and $G$ vanish at $(0,0)$.

As another result in the same direction, we obtain easily from Theorem 8 that an effectively asymptotic version of Statement (a) and (b) in Theorem 8 holds, merely assuming that $g_{1}, \ldots, g_{n}$ are multiplicatively independent modulo $\mathbf{k}$; here the effectivity means that we have an effective lower bound for $\ell$ in the following statement.

Theorem 11. Let $F, G \in K\left[x_{1}, \ldots, x_{n}\right]$ be nonconstant coprime polynomials. Let $g_{1}, \ldots, g_{n} \in K^{*}$, not all constant. Then for any $\epsilon>0$, there exist an integer $m$ and constant $c_{1}$ and $c_{2}$ depending only on $\epsilon$, such that for each positive integer

$$
\ell>c_{1}(\tilde{h}(F)+\tilde{h}(G))+c_{2}\left(\mathfrak{g}+n \max _{1 \leq i \leq n}\left\{h\left(g_{i}\right)\right\}\right),
$$

either we have $g_{1}^{m_{1}} \cdots g_{n}^{m_{n}} \in \mathbf{k}$ for some integers $m_{1}, \ldots, m_{n}$, not all zeros with $\sum\left|m_{i}\right| \leq 2 m$, or the following two statements hold.

(a) $N_{S, g c d}\left(F\left(g_{1}^{\ell}, \ldots, g_{n}^{\ell}\right), G\left(g_{1}^{\ell}, \ldots, g_{n}^{\ell}\right)\right) \leq \epsilon \max _{1 \leq i \leq n} h\left(g_{i}^{\ell}\right)$;

(b) $h_{\mathrm{gcd}}\left(F\left(g_{1}^{\ell}, \ldots, g_{n}^{\ell}\right), G\left(g_{1}^{\ell}, \ldots, g_{n}^{\ell}\right)\right) \leq \epsilon \max _{1 \leq i \leq n} h\left(g_{i}^{\ell}\right)$, if we further assume that not both of $F$ and $G$ vanish at $(0, \ldots, 0)$. 
Remark 12. When $F, G \in \mathbb{C}\left[x_{1}, \ldots, x_{n}\right]$ be a coprime pair of nonconstant polynomials and $g_{1}, \ldots, g_{n} \in \mathbb{C}[z]$ are multiplicatively independent modulo $\mathbb{C}$, then the results in 8 also imply Statement (a) and (b) in Theorem 11. Our statement here is stronger since we have formulated effective bounds on $\ell$ and the $m_{i}$ such that $g_{1}^{m_{1}} \cdots g_{n}^{m_{n}} \in \mathbf{k}$. When $n>2$, the only other previous result in this direction appears to be a result of Ostafe [9, Th. 1.3], which considers special polynomials such as $F=x_{1} \cdots x_{r}-1, G=x_{r+1} \cdots x_{n}-1$, but proves a uniform bound in place of Statement (a) and (b) independent of $\ell$. In the case where $n=2$, previous results include the original theorem of Ailon-Rudnick [1] in this setting, i.e. $F=x_{1}-1, G=x_{2}-1$, and extensions of Ostafe 9 and Pakovich and Shparlinski [10] (all with uniform bounds). It is noted in [9] that it appears to be difficult to extend the techniques used there to obtain results for general $F$ and $G$.

We collect the background materials in Section 22 We will prove some main lemmas in Section 3 , The proofs of Theorem 2 and Theorem 6 are given in Section 5 and Section 4 respectively. Finally, we establish the gcd theorems in Section 6.

\section{Preliminaries}

Recall that $K$ is the function field of the smooth projective curve $C$ of genus $\mathfrak{g}$ defined over the algebraically closed field $\mathbf{k}$ of characteristic zero. At each point $\mathbf{p} \in C(\mathbf{k})$, we may choose a uniformizer $t_{\mathbf{p}}$ to define a normalized order function $v_{\mathbf{p}}:=\operatorname{ord}_{\mathbf{p}}: K \rightarrow \mathbb{Z} \cup\{+\infty\}$. Let $S \subset C(\mathbf{k})$ be a finite subset. We denote the ring of $S$-integers in $K$ and the group of $S$-units in $K$ respectively by

$$
\mathcal{O}_{S}:=\left\{f \in K \mid v_{\mathbf{p}}(f) \geq 0 \text { for all } \mathbf{p} \notin S\right\},
$$

and

$$
\mathcal{O}_{S}^{*}:=\left\{f \in K \mid v_{\mathbf{p}}(f)=0 \text { for all } \mathbf{p} \notin S\right\} .
$$

For simplicity of notation, for $f \in K^{*}$ and $\mathbf{p} \in C(\mathbf{k})$ we let

$$
v_{\mathbf{p}}^{0}(f):=\max \left\{0, v_{\mathbf{p}}(f)\right\}, \quad \bar{v}_{\mathbf{p}}^{0}(f):=\min \left\{1, v_{\mathbf{p}}^{0}(f)\right\},
$$

i.e. its order of zero at $\mathbf{p}$ and its truncated value;

$$
v_{\mathbf{p}}^{\infty}(f):=-\min \left\{0, v_{\mathbf{p}}(f)\right\}, \quad \bar{v}_{\mathbf{p}}^{\infty}(f):=\min \left\{1, v_{\mathbf{p}}^{\infty}(f)\right\},
$$

i.e. its order of pole at $\mathbf{p}$ and its truncated value. The height of $f$ is defined by

$$
h(f):=\sum_{\mathbf{p} \in C}-v_{\mathbf{p}}^{\infty}(f),
$$

which counts the number of poles of $f$ with multiplicities. For any $\mathbf{f}:=\left[f_{0}: \cdots: f_{m}\right] \in \mathbb{P}^{m}(K)$ with $m \geq 1$ and $f_{0}, \ldots, f_{m} \in K$, we define $v_{\mathbf{p}}(\mathbf{f}):=\min \left\{v_{\mathbf{p}}\left(f_{0}\right), \ldots, v_{\mathbf{p}}\left(f_{m}\right)\right\}$ and

$$
h(\mathbf{f})=h\left(f_{0}, \ldots, f_{m}\right):=\sum_{\mathbf{p} \in C}-v_{\mathbf{p}}(\mathbf{f}) .
$$

For a finite subset $S$ of $C$ and $f \in K^{*}$, we let

$$
\bar{N}_{S}(f)=\sum_{\mathbf{p} \in C \backslash S} \bar{v}_{\mathbf{p}}^{0}(f) .
$$


be the cardinality of the set of zeros of $f$ outside $S$; and

$$
N_{S}(f)=\sum_{\mathbf{p} \notin S} v_{\mathbf{p}}^{0}(f)
$$

be the number of the zero, counting multiplicities, of $f$ outside of $S$. For any $f, g \in K$, we let

$$
N_{S, \operatorname{gcd}}(f, g):=\sum_{\mathbf{p} \in C \backslash S} \min \left\{v_{\mathbf{p}}^{0}(f), v_{\mathbf{p}}^{0}(g)\right\}
$$

and

$$
h_{\mathrm{gcd}}(f, g):=\sum_{\mathbf{p} \in C} \min \left\{v_{\mathbf{p}}^{0}(f), v_{\mathbf{p}}^{0}(g)\right\} .
$$

Let $\mathbf{x}:=\left(x_{1}, \ldots, x_{n}\right)$ be a tuple of $n$ variables, and $F=\sum_{\mathbf{i} \in I_{F}} a_{\mathbf{i}} \mathbf{x}^{\mathbf{i}} \in K[\mathbf{x}]$ be a nonzero polynomial, where $I_{F}$ is the (nonempty finite) set of those indices $\mathbf{i}=\left(i_{1}, \ldots, i_{n}\right)$ with $a_{\mathbf{i}} \neq 0$; here, each $i_{j}$ is a nonnegative integer, and we put $\mathbf{x}^{\mathbf{i}}:=x_{1}^{i_{1}} \cdots x_{n}^{i_{n}}$. We define the height $h(F)$ and the relevant height $\tilde{h}(F)$ as follows. Put

$$
v_{\mathbf{p}}(F):=\min _{\mathbf{i} \in I_{F}}\left\{v_{\mathbf{p}}\left(a_{\mathbf{i}}\right)\right\} \quad \text { for } \mathbf{p} \in C .
$$

and define

$$
\begin{gathered}
h(F):=\sum_{\mathbf{p} \in C}-v_{\mathbf{p}}(F), \\
\tilde{h}(F):=\sum_{\mathbf{p} \in C}-\min \left\{0, v_{\mathbf{p}}(F)\right\} .
\end{gathered}
$$

Notice that Gauss's lemma can be stated as

$$
v_{\mathbf{p}}(F G)=v_{\mathbf{p}}(F)+v_{\mathbf{p}}(G),
$$

where $F$ and $G$ are in $K\left[x_{1}, \ldots, x_{n}\right]$ and $\mathbf{p} \in C$. Consequently, we have that

$$
h(F G)=h(F)+h(G) .
$$

Although relevant height $\tilde{h}(F)$ is not projectively invariant, it suits better when comparing with the height of an individual coefficient of $F$. Indeed, we have from the definitions that

$$
h\left(a_{\mathbf{i}}\right) \leq \tilde{h}(F) \quad \text { and } \quad \tilde{h}\left(a_{\mathbf{i}}^{-1} F\right)=h\left(a_{\mathbf{i}}^{-1} F\right)=h(F) \leq \tilde{h}(F),
$$

where $a_{\mathbf{i}}$ is any non-zero coefficient $F$.

We now recall the definitions of global and local derivations on $K$. Let $t \in K \backslash \mathbf{k}$, which will be fixed later. The mapping $g \rightarrow \frac{d g}{d t}$ on $\mathbf{k}(t)$, formal differentiation on $\mathbf{k}(t)$ with respect to $t$, extends uniquely to a global derivation on $K$ as $K$ is a finite separable extension of $\mathbf{k}(t)$. Furthermore, since an element in $K$ can be written as a Laurent series in $t_{\mathbf{p}}$, the local derivative of $\eta \in K$ with respect to $t_{\mathbf{p}}$, denoted by $d_{\mathbf{p}} \eta:=\frac{d \eta}{d t_{\mathbf{p}}}$, is given by the formal differentiation on $\mathbf{k}\left(\left(t_{\mathbf{p}}\right)\right)$ with respect to $t_{\mathbf{p}}$. Consequently,

$$
\frac{d \eta}{d t}=d_{\mathbf{p}} \eta \cdot\left(d_{\mathbf{p}} t\right)^{-1}
$$


The following results are consequences of the Riemann-Roch Theorem. We refer to [2, Corollary 7] for a proof.

Proposition 13. For each point $\mathbf{p}_{\infty} \in C$, we can find some $t \in K \backslash \mathbf{k}$ satisfying the following conditions:

(a) $t$ has exactly one pole at $\mathbf{p}_{\infty}$;

(b) $h(t) \leq \mathfrak{g}+1$;

(c) $\sum_{\mathbf{p} \in C} v_{\mathbf{p}}^{0}\left(d_{\mathbf{p}} t\right) \leq 3 \mathfrak{g}$.

We will use the following result of Brownawell-Masser 3 .

Theorem 14. Let the characteristic of $K$ be zero. If $f_{1}, \ldots, f_{n} \in \mathcal{O}_{S}^{*}$ and $f_{1}+\cdots+f_{n}=1$, then either some proper subsum of $f_{1}+\cdots+f_{n}$ vanishes or

$$
\max _{1 \leq i \leq n} h\left(f_{i}\right) \leq \frac{n(n-1)}{2} \max \{0,2 \mathfrak{g}-2+|S|\}
$$

The following is an analogue of Green's lemma in Nevanlinna's theory.

Corollary 15. Let the characteristic of $K$ be zero and $\ell$ be an integer. Let $a_{1}, \ldots, a_{n}, f_{1}, \ldots, f_{n} \in K^{*}$, $n \geq 2$. If

$$
a_{1} f_{1}^{\ell}+\cdots+a_{n} f_{n}^{\ell}=0
$$

and no proper subsum of $a_{1} f_{1}^{\ell}+\cdots+a_{n} f_{n}^{\ell}$ vanishes. Then $\frac{f_{i}}{f_{j}} \in \mathbf{k}$, for all $1 \leq i, j \leq n$, if

$$
\ell>(n-1)^{2}(n-2) \max \{1, \mathfrak{g}\}+(n-1)^{4} h\left(a_{1}, \ldots, a_{n}\right) .
$$

Proof. Let $b_{i}=\frac{a_{i}}{a_{n}}$ and $g_{i}=\frac{f_{i}}{f_{n}}$. Then

$$
b_{1} g_{1}^{\ell}+\cdots+b_{n-1} g_{n-1}^{\ell}=-1,
$$

and no proper subsum of $b_{1} g_{1}^{\ell}+\cdots+b_{n-1} g_{n-1}^{\ell}$ vanishes. Suppose that (2.4) holds and that at least one of the $g_{i}, 1 \leq i \leq n-1$, is not constant. Let $S$ be the set consisting of the zeros and poles of $b_{i}$ and $g_{i}$, $1 \leq i \leq n-1$. Then

$$
2 \leq|S| \leq 2 \sum_{i=1}^{n-1}\left(h\left(b_{i}\right)+h\left(g_{i}\right)\right),
$$

and all the $b_{i}$ and $g_{i}$ are $S$-units. Applying Theorem 14 to the equation (2.5), we have

$$
h\left(b_{i} g_{i}^{\ell}\right) \leq \frac{(n-1)(n-2)}{2}\left(2 \mathfrak{g}-2+2 \sum_{j=1}^{n-1}\left(h\left(b_{j}\right)+h\left(g_{j}\right)\right)\right),
$$

for $1 \leq i \leq n-1$. As

$$
\ell h\left(g_{i}\right) \leq h\left(b_{i} g_{i}^{\ell}\right)+h\left(b_{i}\right),
$$

for $1 \leq i \leq n-1$, together with (2.6) we have

$$
\ell \sum_{i=1}^{n-1} h\left(g_{i}\right) \leq \sum_{i=1}^{n-1} h\left(b_{i}\right)+(n-1)^{2}(n-2)\left(\mathfrak{g}-1+\sum_{i=1}^{n-1}\left(h\left(b_{i}\right)+h\left(g_{i}\right)\right)\right) .
$$


Hence,

$$
\left(\ell-(n-1)^{2}(n-2)\right) \sum_{i=1}^{n-1} h\left(g_{i}\right) \leq(n-1)^{2}(n-2)(\mathfrak{g}-1)+\left((n-1)^{2}(n-2)+1\right) \sum_{i=1}^{n-1} h\left(b_{i}\right) .
$$

Since one of the $g_{i}$ is not constant, $\ell>(n-1)^{2}(n-2)$ by (2.4) and $h\left(b_{i}\right)=h\left(a_{i}, a_{n}\right) \leq h\left(a_{1}, \ldots, a_{n}\right)$, it implies that

$$
\ell-(n-1)^{2}(n-2) \leq(n-1)^{2}(n-2)(\mathfrak{g}-1)+(n-1)^{4} h\left(a_{1}, \ldots, a_{n}\right),
$$

contradicting to (2.4).

\section{Main Lemmas}

From now on, we will fix a $t$ satisfying the conditions in Proposition 13 and use the notation $\eta^{\prime}:=\frac{d \eta}{d t}$ for $\eta \in K$. We will use the follow estimate.

Lemma 16. Let $S$ be a finite subset of $C$. Then the following statements hold.

(a) $N_{S, g c d}\left(\eta, \eta^{\prime}\right) \geq N_{S}(\eta)-\bar{N}_{S}(\eta)-3 \mathfrak{g}$ for any $\eta \in K$.

(b) $h\left(1, \frac{\eta_{1}^{\prime}}{\eta_{1}}, \ldots, \frac{\eta_{\ell}^{\prime}}{\eta_{\ell}}\right) \leq|S|+3 \mathfrak{g}$, where $\eta_{i} \in \mathcal{O}_{S}^{*}$ for each $1 \leq i \leq \ell$.

Proof. It is clear from (2.3) that

$$
\begin{array}{ll}
v_{\mathbf{p}}\left(\eta^{\prime}\right)=v_{\mathbf{p}}(\eta)-1-v\left(d_{\mathbf{p}} t\right) & \text { if } v_{\mathbf{p}}(\eta) \neq 0 \\
v_{\mathbf{p}}\left(\eta^{\prime}\right) \geq-v\left(d_{\mathbf{p}} t\right) & \text { if } v_{\mathbf{p}}(\eta)=0 .
\end{array}
$$

Consequently,

$$
\begin{aligned}
N_{S, \operatorname{gcd}}\left(\eta, \eta^{\prime}\right) & =\sum_{\mathbf{p} \notin S} \min \left\{v_{\mathbf{p}}^{0}(\eta), v_{\mathbf{p}}^{0}\left(\eta^{\prime}\right)\right\} \\
& =\sum_{v_{\mathbf{p}}(\eta)>0, \mathbf{p} \notin S} \min \left\{v_{\mathbf{p}}(\eta), v_{\mathbf{p}}(\eta)-1-v\left(d_{\mathbf{p}} t\right)\right\} \\
& \geq \sum_{v_{\mathbf{p}}(\eta)>0, \mathbf{p} \notin S}\left(v_{\mathbf{p}}(\eta)-1-v^{0}\left(d_{\mathbf{p}} t\right)\right) \\
& \geq N_{S}(\eta)-\bar{N}_{S}(\eta)-3 \mathfrak{g}
\end{aligned}
$$

by Proposition 13 (c). Again by (3.1) and the assumption that $\eta_{i} \in \mathcal{O}_{S}^{*}$ for each $1 \leq i \leq \ell$, we have

$$
\begin{aligned}
h\left(1, \frac{\eta_{1}^{\prime}}{\eta_{1}}, \ldots, \frac{\eta_{\ell}^{\prime}}{\eta_{\ell}}\right) & =\sum_{\mathbf{p} \in C}-\min _{1 \leq i \leq \ell}\left\{0, v_{\mathbf{p}}\left(\eta_{i}^{\prime}\right)-v_{\mathbf{p}}\left(\eta_{i}\right)\right\} \\
& \leq \sum_{\mathbf{p} \in S}-\min \left\{0,-1-v\left(d_{\mathbf{p}} t\right)\right\}+\sum_{\mathbf{p} \in C \backslash S}-\min \left\{0,-v\left(d_{\mathbf{p}} t\right)\right\} \\
& \leq|S|+\sum_{\mathbf{p} \in C} v^{0}\left(d_{\mathbf{p}} t\right) \\
& \leq|S|+3 \mathfrak{g}
\end{aligned}
$$

by Proposition 13 (c). 
For convenience of discussion, we will use the following convention. Let $\mathbf{i}=\left(i_{1}, \ldots, i_{n}\right) \in \mathbb{Z}^{n}$ and $\mathbf{u}=\left(u_{1}, \ldots, u_{n}\right) \in\left(K^{*}\right)^{n}$. We denote by $\mathbf{x}:=\left(x_{1}, \ldots, x_{n}\right), \mathbf{x}^{\mathbf{i}}:=x_{1}^{i_{1}} \cdots x_{n}^{i_{n}}, \mathbf{u}^{\mathbf{i}}:=u_{1}^{i} \cdots u_{n}^{i_{n}} \in K^{*}$ and $|\mathbf{i}|:=\sum_{j=1}^{n}\left|i_{j}\right|$. For a polynomial $F(\mathbf{x})=\sum_{\mathbf{i}} a_{\mathbf{i}} \mathbf{x}^{\mathbf{i}} \in K\left[x_{1}, \ldots, x_{n}\right]$, we denote by $I_{F}$ the set of exponents $\mathbf{i}$ such that $a_{\mathbf{i}} \neq 0$ in the expression of $F$, and define

$$
D_{\mathbf{u}}(F)(\mathbf{x}):=\sum_{\mathbf{i} \in I_{F}} \frac{\left(a_{\mathbf{i}} \mathbf{u}^{\mathbf{i}}\right)^{\prime}}{\mathbf{u}^{\mathbf{i}}} \mathbf{x}^{\mathbf{i}}
$$

Clearly, we have

$$
F(\mathbf{u})^{\prime}=D_{\mathbf{u}}(F)(\mathbf{u})
$$

and the following product rule:

$$
D_{\mathbf{u}}(F G)=D_{\mathbf{u}}(F) G+F D_{\mathbf{u}}(G)
$$

for each $F, G \in K\left[x_{1}, \ldots, x_{n}\right]$.

The following proposition gives an upper bound on height of the coefficients of $D_{\mathbf{u}}(F)$ when the $u_{i}$ 's are $S$-units. This is a crucial step.

Proposition 17. Let $F$ be a nonconstant polynomial in $K\left[x_{1}, \ldots, x_{n}\right]$ and $\mathbf{u}=\left(u_{1}, \ldots, u_{n}\right) \in\left(O_{S}^{*}\right)^{n}$. Then there exist $c_{1}, c_{2}$ depending only on $\operatorname{deg} F$ such that

$$
\tilde{h}\left(D_{\mathbf{u}}(F)\right) \leq c_{1} \tilde{h}(F)+c_{2} \max \{1,2 \mathfrak{g}-2+|S|\} .
$$

Proof. Let $F\left(x_{1}, \ldots, x_{n}\right)=\sum_{\mathbf{i} \in I_{F}} a_{\mathbf{i}} \mathbf{x}^{\mathbf{i}}$. We then choose $S^{\prime}$ containing $S$ and all the zeros and poles of all $a_{\mathbf{i}}$ for $\mathbf{i} \in I_{F}$. Then

$$
\left|S^{\prime}\right| \leq|S|+2 \sum_{\mathbf{i} \in I_{F}} h\left(a_{\mathbf{i}}\right) \leq|S|+2\left|I_{F}\right| \tilde{h}(F)
$$

and $a_{\mathbf{i}} \in O_{S^{\prime}}^{*}$ for each $\mathbf{i} \in I_{F}$. As

$$
D_{\mathbf{u}}(F)(\mathbf{x})=\sum_{\mathbf{i} \in I_{F}} a_{\mathbf{i}} \cdot \frac{\left(a_{\mathbf{i}} \mathbf{u}^{\mathbf{i}}\right)^{\prime}}{a_{\mathbf{i}} \mathbf{u}^{\mathbf{i}}} \mathbf{x}^{\mathbf{i}}
$$

we have that

$$
\begin{aligned}
\tilde{h}\left(D_{\mathbf{u}}(F)\right) & \leq h\left(1,\left(a_{\mathbf{i}}\right)_{\mathbf{i} \in I_{F}}\right)+h\left(1,\left(\frac{\left(a_{\mathbf{i}} \mathbf{u}^{\mathbf{i}}\right)^{\prime}}{a_{\mathbf{i}} \mathbf{u}^{\mathbf{i}}}\right)_{\mathbf{i} \in I_{F}}\right) \\
& \leq|S|+\left(2\left|I_{F}\right|+1\right) \tilde{h}(F)+3 \mathfrak{g} .
\end{aligned}
$$

by Lemma 16 and (3.5). The assertion is now clear since $\left|I_{F}\right| \leq(\underset{n}{n+\operatorname{deg} F})$ and $|S|+3 \mathfrak{g} \leq 3 \max \{1,2 \mathfrak{g}-2+$ $|S|\}$.

Lemma 18. For any irreducible $F(\mathbf{x})=\sum_{\mathbf{i} \in I_{F}} a_{\mathbf{i}} \mathbf{x}^{\mathbf{i}} \in K\left[x_{1}, \ldots, x_{n}\right]$ and $\mathbf{u} \in\left(K^{*}\right)^{n}$, the two polynomials $F$ and $D_{\mathbf{u}}(F)$ are not coprime if and only if $\frac{a_{\mathbf{i}} \mathbf{u}^{\mathbf{i}}}{a_{\mathbf{j}} \mathbf{u}^{\mathbf{j}}} \in \mathbf{k}^{*}$ whenever $\mathbf{i}, \mathbf{j} \in I_{F}$.

Proof. It is clear from (3.2) that $\operatorname{deg} D_{\mathbf{u}}(F) \leq \operatorname{deg} F$ for each $j$. Since $F$ is irreducible, it follows that $F$ and $D_{\mathbf{u}}(F)$ are not coprime if and only if $D_{\mathbf{u}}(F)=\lambda F$ for some $\lambda \in K$, i.e., $\frac{\left(a_{\mathbf{i}} \mathbf{u}^{\mathbf{i}}\right)^{\prime}}{\mathbf{u}^{\mathbf{i}}}=\lambda a_{\mathbf{i}}$ for each $\mathbf{i}$. The latter 
condition is equivalent to that for those $\mathbf{i}, \mathbf{j} \in I_{F}$ we must have $\frac{\left(a_{\mathbf{i}} \mathbf{u}^{\mathbf{i}}\right)^{\prime}}{a_{\mathbf{i}} \mathbf{u}^{\mathbf{i}}}=\frac{\left(a_{\mathbf{j}} \mathbf{u}^{\mathbf{j}}\right)^{\prime}}{a_{\mathbf{j}} \mathbf{u}^{\mathbf{j}}}$, which is equivalent to that $\left(\frac{a_{\mathrm{i}} \mathbf{u}^{\mathbf{i}}}{a_{\mathrm{j}} \mathbf{u}^{\mathrm{j}}}\right)^{\prime}=0$.

Lemma 19. Let $F=\prod_{i=1}^{r} P_{i} \in K\left[x_{1}, \ldots, x_{n}\right]$, where $P_{i}, 1 \leq i \leq r$, is irreducible and not monomial in $K\left[x_{1}, \ldots, x_{n}\right]$. Let $\mathbf{u} \in\left(K^{*}\right)^{n}$, $\mathbf{e}=\left(e_{1}, \ldots, e_{r}\right)$ be an $r$-tuple of positive integers. Then either the two polynomials $F$ and

$$
F_{\mathbf{e}, \mathbf{u}}:=\sum_{i=1}^{r} e_{i} D_{\mathbf{u}}\left(P_{i}\right) \prod_{j \neq i} P_{j}
$$

are coprime in $K\left[x_{1}, \ldots, x_{n}\right]$ or

$$
h\left(u_{1}^{m_{1}} \cdots u_{n}^{m_{n}}\right) \leq h(F)
$$

for some $\left(m_{1}, \ldots m_{n}\right) \in \mathbb{Z}^{n} \backslash\{(0, \ldots, 0)\}$ with $\sum\left|m_{i}\right| \leq 2 \operatorname{deg} F$.

Proof. If $F$ and $F_{\mathbf{e}, \mathbf{u}}$ are not coprime in $K\left[x_{1}, \ldots, x_{n}\right]$, some $P_{i}$ must divide $F_{\mathbf{e}, \mathbf{u}}$ and thus divide $D_{\mathbf{u}}\left(P_{i}\right)$. Since $P_{i}$ is not a monomial, we have $P_{i}=\sum_{\mathbf{i} \in I_{P_{i}}} a_{\mathbf{i}} \mathbf{x}^{\mathbf{i}}$ with $\left|I_{P_{i}}\right| \geq 2$. Then Lemma 18 implies that $\frac{a_{\mathbf{i}} \mathbf{u}^{\mathbf{i}}}{a_{\mathbf{j}} \mathbf{u}^{\mathbf{j}}} \in \mathbf{k}^{*}$ whenever $\mathbf{i}, \mathbf{j} \in I_{P_{i}}$. Since $\left|I_{P_{i}}\right| \geq 2$, we can choose distinct $\mathbf{i}, \mathbf{j} \in I_{P_{i}}$. Thus

$$
h\left(\mathbf{u}^{\mathbf{i}-\mathbf{j}}\right)=h\left(a_{\mathbf{i}}^{-1} a_{\mathbf{j}}\right) \leq h\left(P_{i}\right) \leq h(F)
$$

by (2.1) with $0 \neq|\mathbf{i}-\mathbf{j}| \leq 2 \operatorname{deg} P_{i} \leq 2 \operatorname{deg} F$.

Lemma 20. Let $d \geq 2$ be an integer, $F_{1}, \ldots, F_{r} \in O_{S}\left[x_{1}, \ldots, x_{n}\right]$ be distinct non-monomial polynomials which are irreducible in $K\left[x_{1}, \ldots, x_{n}\right]$, and put $F:=F_{1}^{e_{1}} \cdots F_{r}^{e_{r}}$ with $1 \leq e_{i}<d$ for each $i$. Let $\mathbf{u}=$ $\left(u_{1}, \ldots, u_{n}\right) \in\left(\mathcal{O}_{S}^{*}\right)^{n}$. If $F(\mathbf{u})=g^{d}$ for some $g \in K^{*}$, then for every $\varepsilon>0$ there exist an integer $m$ and reals $c_{1}, c_{2}$, all depending only on $(\varepsilon, \delta, d)$, where $\operatorname{deg} F \leq \delta$, such that either

$$
N_{S}(F(\mathbf{u})) \leq \varepsilon \max _{1 \leq j \leq n}\left\{h\left(u_{j}\right)\right\}
$$

or

$$
h\left(u_{1}^{m_{1}} \cdots u_{n}^{m_{n}}\right) \leq c_{1} \tilde{h}(F)+c_{2} \max \{1,2 \mathfrak{g}-2+|S|\}
$$

for some integers $m_{1}, \ldots, m_{n}$ not all zeros with $\sum\left|m_{i}\right| \leq 2 m$.

Proof. By (3.3) and the product rule (3.4) of $D_{\mathbf{u}}$, we have

$$
d g^{d-1} g^{\prime}=D_{\mathbf{u}}(F)(\mathbf{u})=\left(F_{1}^{e_{1}-1}(\mathbf{u}) \cdots F_{r}^{e_{r}-1}(\mathbf{u})\right) F_{\mathbf{e}, \mathbf{u}}(\mathbf{u}),
$$

where $F_{\mathbf{e}, \mathbf{u}}:=\sum_{i=1}^{r} e_{i} D_{\mathbf{u}}\left(P_{i}\right) \prod_{j \neq i} P_{j}$ as defined in Lemma 19, from which it follows that either $\bar{F}:=$ $F_{1} \cdots F_{r}$ and $F_{\mathbf{e}, \mathbf{u}}$ are coprime in $K\left[x_{1}, \ldots, x_{n}\right]$ or the second assertion (3.8) holds with any $\left(c_{1}, c_{2}, m\right)$ with $c_{1} \geq 1, c_{2} \geq 0$ and $m \geq \delta$. It remains to consider the case where the former condition holds. By Theorem 8 and Proposition 17, for any $\epsilon^{\prime}>0$ there exist an integer $m \geq \delta$, positive reals $c_{i}, 1 \leq i \leq 4$, with $c_{1} \geq 1$ and $c_{2} \geq 0$, depending only on $\epsilon^{\prime}$, such that whenever

$$
\max _{1 \leq i \leq n} h\left(u_{i}\right) \geq c_{3} \tilde{h}(F)+c_{4} \max \{1,2 \mathfrak{g}-2+|S|\}
$$


we have either

$$
h\left(u_{1}^{m_{1}} \cdots u_{n}^{m_{n}}\right) \leq c_{1} \tilde{h}(F)+c_{2} \max \{1,2 \mathfrak{g}-2+|S|\}
$$

for some integers $m_{1}, \ldots, m_{n}$, not all zeros with $\sum\left|m_{i}\right| \leq 2 m$, or

$$
N_{S, \operatorname{gcd}}\left(\bar{F}(\mathbf{u}), F_{\mathbf{e}, \mathbf{u}}(\mathbf{u})\right) \leq \epsilon^{\prime} \max _{1 \leq i \leq n} h\left(u_{i}\right)
$$

We note that $h\left(u_{1}^{m_{1}} \cdots u_{n}^{m_{n}}\right) \leq\left(\sum_{1 \leq i \leq n}\left|m_{i}\right|\right) \max _{1 \leq i \leq n} h\left(u_{i}\right)$, which shows that the case where (3.10) does not hold leads to (3.8) once we enlarge $c_{1}$ and $c_{2}$.

If (3.11) holds, then together with (3.9), we have

$$
N_{S, \operatorname{gcd}}\left(F(\mathbf{u}), D_{\mathbf{u}}(F)(\mathbf{u})\right) \leq \sum_{i=1}^{r}\left(e_{i}-1\right) N_{S}\left(F_{i}(\mathbf{u})\right)+\varepsilon^{\prime} \max _{1 \leq j \leq n}\left\{h\left(u_{j}\right)\right\} .
$$

On the other hand, since $g^{d}=F(\mathbf{u})$, the key equality (3.3) and Lemma 16 imply that

$$
N_{S, \operatorname{gcd}}\left(F(\mathbf{u}), D_{\mathbf{u}}(F)(\mathbf{u})\right)=N_{S, \operatorname{gcd}}\left(g^{d},\left(g^{d}\right)^{\prime}\right) \geq(d-1) N_{S}(g)-3 \mathfrak{g} ;
$$

together with the fact $F(\mathbf{u}), F_{1}(\mathbf{u}), \ldots, F_{r}(\mathbf{u}) \in O_{S}$, this gives

$$
N_{S, \operatorname{gcd}}\left(F(\mathbf{u}), D_{\mathbf{u}}(F)(\mathbf{u})\right)+3 \mathfrak{g} \geq \frac{d-1}{d} N_{S}(F(\mathbf{u}))=\frac{d-1}{d} \sum_{i=1}^{r} e_{i} N_{S}(F(\mathbf{u})) .
$$

Together with (3.12), we have

$$
\sum_{i=1}^{k}\left(1-\frac{e_{i}}{d}\right) N_{S}\left(F_{i}(\mathbf{u})\right) \leq 2 \varepsilon^{\prime} \max _{1 \leq j \leq n}\left\{h\left(u_{j}\right)\right\}
$$

by further requiring that $3 \mathfrak{g} \leq \varepsilon^{\prime} \max _{1 \leq j \leq n}\left\{h\left(u_{j}\right)\right\}$; this is possible since we may assume that $c_{4} \geq 3 \mathfrak{g} / \epsilon^{\prime}$ in (3.10). Since $e_{i}<d$ and $N_{S}\left(F_{i}(\mathbf{u})\right) \geq 0$ for each $i$, it implies that

$$
\frac{1}{d} N_{S}\left(F_{i}(\mathbf{u})\right) \leq 2 \varepsilon^{\prime} \max _{1 \leq j \leq n}\left\{h\left(u_{j}\right)\right\}
$$

for each $i$. By taking $\varepsilon^{\prime}=\frac{\varepsilon}{2 d \delta} \leq \frac{\varepsilon}{2 d \operatorname{deg} F} \leq \frac{\varepsilon}{2 d\left(e_{1}+\cdots+e_{r}\right)}$, we have

$$
N_{S}(F(\mathbf{u}))=\sum_{i=1}^{r} e_{i} N_{S}\left(F_{i}(\mathbf{u})\right) \leq \varepsilon \max _{1 \leq j \leq n}\left\{h\left(u_{j}\right)\right\}
$$

\section{Proof of Theorem 6}

For each finite extension $L$ over $K$, denote by $h_{L}$ the height function (both on $L$ and on $L\left[x_{1}, \ldots, x_{n}\right]$ ) obtained from the same construction of $h$ with $K$ replaced by $L$; similar for the notation $\tilde{h}_{L}$, and $O_{L, \widetilde{S}}, N_{L, \widetilde{S}}$, $\bar{N}_{L, \widetilde{S}}$, where $\widetilde{S} \subset C_{L}(\mathbf{k})$ is a finite subset, and $C_{L}$ be a smooth projective curve over $\mathbf{k}$ such that $L=\mathbf{k}\left(C_{L}\right)$. We need the following result from [12, Proposition 2.4]. 
Proposition 21. Let $\alpha$ be a nonconstant algebraic element over $K$ with $[K(\alpha): K]=m$. Denote by $L=K(\alpha)$ and let $C_{L}$ be a smooth projective curve over $\mathbf{k}$ of genus $\mathfrak{g}_{L}$ such that $L=\mathbf{k}\left(C_{L}\right)$. Then

$$
\mathfrak{g}_{L}-1 \leq m(\mathfrak{g}-1)+(m-1) h_{L}(\alpha) .
$$

In the following proof, we will use, without further prompts, the standard fact that $h_{L}(a)=[L: K] h(a)$ for every $a \in K$, and that $\tilde{h}_{L}(P) \leq[L: K] \tilde{h}(P)$ for every $P \in K\left[x_{1}, \ldots, x_{n}\right]$.

Proof of Theorem [6. We may assume $|S| \geq 2$, for otherwise $\mathcal{O}_{S}^{*}=\mathbf{k}^{*}$ and the desired conclusion holds trivially. For each $\ell \in \mathbb{N}$, put $\mathbf{u}^{\ell}:=\left(u_{1}^{\ell}, \ldots, u_{n}^{\ell}\right) \in\left(O_{S}^{*}\right)^{n}$. We may suppose that there is indeed some $\ell \in \mathbb{N}$ with

$$
\ell \geq c_{1} \tilde{h}(F)+c_{2} \max \{1,2 \mathfrak{g}-2+|S|\}
$$

such that $F\left(\mathbf{u}^{\ell}\right)$ is a $d$-th power in $K$, where $c_{1}$ and $c_{2}$ will be determined in the end of the proof.

Fix a total ordering on the set of monomials in $K\left[x_{1}, \ldots, x_{n}\right]$ and say that an element $Q \in K\left[x_{1}, \ldots, x_{n}\right]$ is monic if the coefficient attached to largest monomial appearing in $Q$ with a non-zero coefficient is 1 . Since $F \neq 0$ in $K\left[x_{1}, \ldots, x_{n}\right]$, it follows from our hypothesis that we may write $F=a \mathbf{x}^{\mathbf{i}} G^{d} P$, where $P \in K\left[x_{1}, \ldots, x_{n}\right] \backslash K$ is $d$-th power free monic polynomial with no (non-trivial) monomial factors, $G \in$ $K\left[x_{1}, \ldots, x_{n}\right]$ is monic, $\mathbf{x}^{\mathbf{i}} \in K\left[x_{1}, \ldots, x_{n}\right]$ is a monomial and $a \in K^{*}$. Note that

$$
\tilde{h}(P)=h(P) \leq h(F) \leq \tilde{h}(F),
$$

and $h(a) \leq \tilde{h}(F)$ since $a$ is the coefficients of the largest monomial appearing in $F$.

Write

$$
P=\sum_{\mathbf{i} \in I_{P}} a_{\mathbf{i}} \mathbf{x}^{\mathbf{i}} \quad \text { with each } a_{\mathbf{i}} \text { being nonzero. }
$$

By our setting, we have that $\left|I_{P}\right| \geq 2$. Choose a finite subset $S_{P} \subset C(\mathbf{k})$ containing $S$ such that $a_{\mathbf{i}} \in O_{S_{P}}^{*}$ for each $\mathbf{i} \in I_{P}$, that each monic irreducible factor of $P$ is in $O_{S_{P}}\left[x_{1}, \ldots, x_{n}\right]$, and that by (4.2) we have

$$
2 \leq\left|S_{P}\right| \leq|S|+2\left|I_{P}\right| \tilde{h}(P)+\operatorname{deg} P \cdot\left(\begin{array}{c}
n+\operatorname{deg} P \\
n
\end{array}\right) \tilde{h}(P) \leq|S|+(\operatorname{deg} F+2)\left(\begin{array}{c}
n+\operatorname{deg} F \\
n
\end{array}\right) \tilde{h}(F) .
$$

Let $L:=K(\alpha)$ with some $d$-th root $\alpha$ of $a \mathbf{u}^{\mathrm{i}}$. Since $F\left(\mathbf{u}^{\ell}\right)$ is a $d$-th power in $K$, it follows that $P\left(\mathbf{u}^{\ell}\right)$ is a $d$-th power in $L$. By Proposition 21, $L$ is the function field of a smooth projective algebraic curve $C_{L}$ of genus $\mathfrak{g}_{L}$ defined over $\mathbf{k}$ with

$$
\begin{aligned}
\mathfrak{g}_{L}-1 & \leq[L: K](\mathfrak{g}-1)+([L: K]-1) \frac{[L: K]}{d} h\left(a \mathbf{u}^{i}\right) \\
& \leq[L: K]\left(\mathfrak{g}-1+\tilde{h}(F)+\operatorname{deg} F \max _{1 \leq j \leq n} h\left(u_{j}\right)\right)
\end{aligned}
$$

since $[L: K] \leq d$ and $\alpha^{d}=a \mathbf{u}^{\mathbf{i}}$. Let $\widetilde{S_{P}} \subset C_{L}(\mathbf{k})$ be the the preimage of $S_{P}$ under the natural map $C_{L}(\mathbf{k}) \rightarrow C(\mathbf{k})$. Then

$$
2 \leq\left|\widetilde{S_{P}}\right| \leq[L: K]\left|S_{P}\right|
$$


Now we have that $P \in L\left[x_{1}, \ldots, x_{n}\right]$ is $d$-th power free and has no (non-trivial) monomial factor, that each irreducible factor of $P$ is in $O_{L, \widetilde{S_{P}}}\left[x_{1}, \ldots, x_{n}\right]$, and that $P\left(\mathbf{u}^{\ell}\right)$ is a $d$-th power in $L$. Hence we can apply Lemma 20 with

$$
\varepsilon=\frac{1}{\left(\begin{array}{c}
n+\operatorname{deg} F \\
n
\end{array}\right)^{2} \max _{1 \leq j \leq n} h\left(u_{j}\right)}
$$

and obtain an integer $m^{\prime}$ and constants $c_{1}^{\prime}, c_{2}^{\prime}$ depending only on $(\varepsilon, \operatorname{deg} F, d)$ such that we have either that

$$
N_{L, \widetilde{S_{P}}}\left(P\left(\mathbf{u}^{\ell}\right)\right) \leq \varepsilon \ell \max _{1 \leq j \leq n} h_{L}\left(u_{j}\right)=\frac{\ell[L: K]}{\left(\begin{array}{c}
n+\operatorname{deg} F \\
n
\end{array}\right)^{2}}
$$

or that

$$
h_{L}\left(u_{1}^{\ell m_{1}} \cdots u_{n}^{\ell m_{n}}\right) \leq c_{1}^{\prime} \tilde{h}_{L}(P)+c_{2}^{\prime} \max \left\{1,2 \mathfrak{g}_{L}-2+\left|\widetilde{S_{P}}\right|\right\}
$$

for some integers $m_{1}, \ldots, m_{n}$, not all zeros with $\sum\left|m_{i}\right| \leq 2 m^{\prime}$. First consider the case where (4.9) holds; thus by (4.4), (4.2), (4.5) and (4.6), we obtain

$h\left(u_{1}^{\ell m_{1}} \cdots u_{n}^{\ell m_{n}}\right) \leq\left(c_{1}^{\prime}+c_{2}^{\prime}(\operatorname{deg} F+2)\left(\begin{array}{c}n+\operatorname{deg} F \\ n\end{array}\right)\right) \tilde{h}(F)+c_{2}^{\prime} \max \left\{1,2 \mathfrak{g}-2+2 \tilde{h}(F)+2 \operatorname{deg} F \max _{1 \leq j \leq n} h\left(u_{j}\right)+|S|\right\}$.

If $u_{1}^{m_{1}} \cdots u_{n}^{m_{n}} \in K \backslash \mathbf{k}$, then $h\left(u_{1}^{\ell m_{1}} \cdots u_{n}^{\ell m_{n}}\right) \geq \ell$ and we would get a contradiction to (4.9), provided

$$
\ell>\left(c_{1}^{\prime}+c_{2}^{\prime}(\operatorname{deg} F+2)\left(\begin{array}{c}
n+\operatorname{deg} F \\
n
\end{array}\right)+2 c_{2}^{\prime}\right) \tilde{h}(F)+c_{2}^{\prime}\left(1+2 \operatorname{deg} F \max _{1 \leq j \leq n} h\left(u_{j}\right)\right) \max \{1,2 \mathfrak{g}-2+|S|\} .
$$

It remains to consider when (4.8) occurs. By (4.3), we have the following equality

$$
P\left(\mathbf{u}^{\ell}\right)=\sum_{\mathbf{i} \in I_{P}} a_{\mathbf{i}} \mathbf{u}^{\mathbf{i} \ell}
$$

First consider the case where the right-hand side of (4.11) has a nontrivial vanishing subsum. (This includes the possibility where $P\left(\mathbf{u}^{\ell}\right)=0$.) In this case, it must have a smallest nontrivial vanishing subsum, i.e., for some $I \subset I_{P}$ (with $|I| \geq 2$ ) we have

$$
\sum_{\mathbf{i} \in I} a_{\mathbf{i}} \mathbf{u}^{\mathbf{i} \ell}=0
$$

Corollary 15$]$ implies that if

$$
\ell>(|I|-1)^{2}(|I|-2) \max \{1, \mathfrak{g}\}+(|I|-1)^{4} h\left(\left[a_{\mathbf{i}}\right]_{\mathbf{i} \in I}\right),
$$

then $\mathbf{u}^{\mathbf{i}-\mathbf{j}} \in \mathbf{k}$ for any distinct $\mathbf{i}, \mathbf{j}$ in $I$. Since $|I| \leq\left|I_{P}\right| \leq\left(\begin{array}{c}n+\operatorname{deg} F \\ n\end{array}\right)$ and $h\left(\left[a_{\mathbf{i}}\right]_{\mathbf{i} \in I}\right) \leq h(P) \leq \tilde{h}(F)$ as well as $\max \{1, \mathfrak{g}\} \leq \max \{1,2 \mathfrak{g}-2+|S|\}$, we see that (4.13) holds if

$$
\ell>\left(\begin{array}{c}
n+\operatorname{deg} F \\
n
\end{array}\right)^{4}(\tilde{h}(F)+\max \{1,2 \mathfrak{g}-2+|S|\}) .
$$

We also note that any distinct $\mathbf{i}, \mathbf{j}$ in $I$ satisfies that $|\mathbf{i}-\mathbf{j}| \leq 2 \operatorname{deg} F$. This settles down the current case. 
It remains to consider the case where the right-hand side of (4.11) has no nontrivial vanishing subsum. Pick some $\mathbf{i}_{0} \in I_{P}$. This case is equivalent to the one where the left-hand side of

$$
\frac{P\left(\mathbf{u}^{\ell}\right)}{a_{\mathbf{i}_{0}} \mathbf{u}^{\mathbf{i}_{0} \ell}}-\sum_{\mathbf{i} \in I_{P} \backslash\left\{\mathbf{i}_{0}\right\}} \frac{a_{\mathbf{i}} \mathbf{u}^{\mathbf{i} \ell}}{a_{\mathbf{i}_{0}} \mathbf{u}^{\mathbf{i}_{0} \ell}}=1
$$

has no nontrivial vanishing subsum. By (4.8), we see that

$$
N_{S_{P}}\left(P\left(\mathbf{u}^{\ell}\right)\right) \leq \frac{\ell}{\left(\begin{array}{c}
n+\operatorname{deg} F \\
n
\end{array}\right)^{2}} .
$$

Let $S_{P, \ell} \subset C(\mathbf{k})$ be a subset containing $S_{P}$ and the zeros of $P\left(\mathbf{u}^{\ell}\right)$ such that

$$
2 \leq\left|S_{P, \ell}\right| \leq\left|S_{P}\right|+\bar{N}_{S_{P}}\left(P\left(\mathbf{u}^{\ell}\right)\right) \leq|S|+(\operatorname{deg} F+2)\left(\begin{array}{c}
n+\operatorname{deg} F \\
n
\end{array}\right) \tilde{h}(F)+\frac{\ell}{\left(\begin{array}{c}
n+\operatorname{deg} F \\
n
\end{array}\right)^{2}}
$$

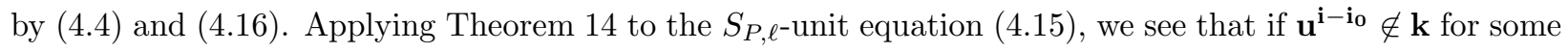
$\mathbf{i} \in I_{P} \backslash\left\{\mathbf{i}_{0}\right\}$, then

$$
\begin{aligned}
\ell \leq h\left(\mathbf{u}^{\left(\mathbf{i}-\mathbf{i}_{\mathbf{o}}\right) \ell}\right) & \leq h\left(\frac{a_{\mathbf{i}} \mathbf{u}^{\mathbf{i} \ell}}{a_{\mathbf{i}_{0}} \mathbf{u}^{\mathbf{i}_{0} \ell}}\right)+h\left(a_{\mathbf{i}}, a_{\mathbf{i}_{0}}\right) \\
& \leq \frac{\left|I_{P}\right|^{2}}{2}\left(2 \mathfrak{g}-2+\left|S_{P, \ell}\right|\right)+h(P) \\
& \leq \frac{\ell}{2}+\left(\frac{1}{2} \operatorname{deg} F+2\right)\left(\begin{array}{c}
n+\operatorname{deg} F \\
n
\end{array}\right)^{3} \tilde{h}(F)+\frac{1}{2}\left(\begin{array}{c}
n+\operatorname{deg} F \\
n
\end{array}\right)^{2} \max \{1,2 \mathfrak{g}-2+|S|\} .
\end{aligned}
$$

Then again, $\mathbf{u}^{\mathbf{i}-\mathbf{i}_{\mathbf{o}}} \in \mathbf{k}$ for every $\mathbf{i} \in I_{P} \backslash\left\{\mathbf{i}_{0}\right\}$, where we note that such $\mathbf{i}$ indeed exists and that $\left|\mathbf{i}-\mathbf{i}_{0}\right| \leq 2 \operatorname{deg} F$, if

$$
\ell>(\operatorname{deg} F+4)\left(\begin{array}{c}
n+\operatorname{deg} F \\
n
\end{array}\right)^{3} \tilde{h}(F)+\left(\begin{array}{c}
n+\operatorname{deg} F \\
n
\end{array}\right)^{2} \max \{1,2 \mathfrak{g}-2+|S|\} .
$$

We obtain the desired conclusion by taking $m:=\max \left\{m^{\prime}, \operatorname{deg} F\right\}$, and choose $c_{1}, c_{2}$ such that (4.1) implies all of (4.10), 4.14) and (4.17).

\section{Proof of Theorem 2}

We need the following result from [6, Proposition 4.2], where it is stated for number fields, but it is clear that the proof works for any field.

Proposition 22. Let $f_{1}, f_{2} \in K\left[x_{0}, x_{1}, \ldots, x_{n}\right] \backslash K\left[x_{0}\right]$ be coprime polynomials. Then, the polynomials $f_{1}(m), f_{2}(m) \in K\left[x_{1}, \ldots, x_{n}\right]$ are coprime for all but perhaps finitely many $m \in \mathbb{N}$.

We also recall the following result of Pasten and the third author on the generalized Büchi's $n$-th power problem.

Theorem 23. [12, Theorem 3] Let $K$ be a function field of a smooth projective curve $C$ of genus $\mathfrak{g}_{K}$ over an algebraically closed field $k$ of characteristic zero. Let $n \geq 2$ and $M$ be integers with

$$
M>4 n \max \{\mathfrak{g}-1,0\}+11 n-3 .
$$


Let $F \in K[x] \backslash \mathbf{k}[x]$ be a monic polynomial of degree $n$. Write $F=P H$ where $P \in \mathbf{k}[x]$ is monic, $H \in K[x]$ is monic and $H$ is not divisible by any non-constant polynomial in $\mathbf{k}[x]$. Let $G_{1}, \ldots, G_{\ell} \in K[x]$ be the distinct monic irreducible factors of $H$ (if any) and let $e_{1}, \ldots, e_{\ell} \geq 1$ be integers such that $H=\prod_{j=1}^{\ell} G_{j}^{e_{j}}$. Let $\mu \geq \max _{j} e_{j}$ be an integer and let $a_{1}, \ldots, a_{M}$ be distinct elements of $\mathbf{k}$.

If for each $1 \leq i \leq M$, the zero multiplicity of those nonzero $F\left(a_{i}\right) \in K^{*}$ at every point $\mathfrak{p} \in C(\mathbf{k})$ is divisible by $\mu$, then $\mu=e_{1}=\cdots=e_{\ell}$ and $H=\left(\prod_{j=1}^{\ell} G_{j}\right)^{\mu}$.

Proof of Theorem 囵. Let $u_{1}, \ldots, u_{n}$ be a (multiplicative) basis of $\Gamma$. Then there exists a Laurent polynomial $f \in K\left[x_{0}, x_{1}, x_{1}^{-1}, \ldots, x_{n}, x_{n}^{-1}\right]$ such that

$$
b(m)=f\left(m, u_{1}^{m}, \ldots, u_{n}^{m}\right) .
$$

We may assume that $f \in K\left[x_{0}, x_{1}, \ldots, x_{n}\right]$ by multiplying $f$ by $\left(x_{1} \cdots x_{n}\right)^{h d}$ for some $h \in \mathbb{N}$ without affecting the assertion. To avoid trivialities, we assume $f$ is not the zero polynomial. We also note that the assumption that $\Gamma \cap k^{*}=\{1\}$ implies that $u_{1}, \ldots, u_{n}$ are multiplicatively independent modulo $\mathbf{k}$.

For each $m \in \mathbb{N}$, it is clear that $\operatorname{deg} f(m, \bullet) \leq \operatorname{deg} f$; since $v_{\mathbf{p}}(a(m)) \geq v_{\mathbf{p}}(a)$ for every $\mathbf{p} \in C(\mathbf{k})$ and nonzero $a \in K\left[x_{0}\right]$, we also see that $\tilde{h}(f(m, \bullet)) \leq \tilde{h}(f)$. Denote by $\mathcal{N}$ the collection of $m \in \mathbb{N}$ such that $b(m)$ is a $d$-th power in $K$, which is an infinite set by the assumption. Thus $f\left(m, u_{1}^{m}, \ldots, u_{n}^{m}\right)$ is a $d$-th power in $K$ for each $m \in \mathcal{N}$. Let $S \subset C(\mathbf{k})$ be a finite subset such that $\mathbf{u}:=\left(u_{1}, \ldots, u_{n}\right) \in\left(\mathcal{O}_{S}^{*}\right)^{n}$. Recall $\mathbf{x}:=\left(x_{1}, \ldots, x_{n}\right)$. Applying Theorem $[$ to each $f(m, \bullet) \in K[\mathbf{x}]$ for each $m \in \mathcal{N}$, we conclude that

$$
f(m, \bullet)=\alpha_{m} \mathbf{x}^{\mathbf{i}_{m}} G_{m}^{d}
$$

for some $\alpha_{m} \in K^{*}$, monomial $\mathbf{x}^{\mathbf{i}_{m}} \in K[\mathbf{x}]$ and $G_{m} \in K[\mathbf{x}]$, provided that $m \in \mathcal{N}$ is sufficiently large.

On the other hand, we factor $f$ in $K\left[x_{0}, \mathbf{x}\right]$ as

$$
f\left(x_{0}, \mathbf{x}\right)=Q\left(x_{0}\right) \mathbf{x}^{\mathrm{i}} \prod_{i=1}^{s} P_{i}\left(x_{0}, \mathbf{x}\right)^{e_{i}}
$$

with some $Q \in K\left[x_{0}\right]$, some monomial $\mathbf{x}^{\mathbf{i}} \in K[\mathbf{x}]$, and some irreducibles $P_{1}, \ldots, P_{s} \in K\left[x_{0}, x_{1}, \ldots, x_{n}\right] \backslash K\left[x_{0}\right]$ without any (non-trivial) monomial factor. Applying Proposition 22 to all $\left(P_{i}, P_{j}\right)$ with $0 \leq i<j \leq s$, where $P_{0}:=x_{1} x_{2} \cdots x_{n}$, we may replace $\mathcal{N}$ by one of its cofinite subset such that for all $m \in \mathcal{N}, 1 \leq i \leq s$ and $1 \leq j \leq s$ with $i \neq j$, we have that $P_{i}(m, \bullet) \in K[\mathbf{x}]$ neither belongs to $K$ nor has (nontrivial) monomial factor, and that $P_{i}(m, \bullet), P_{j}(m, \bullet)$ share no irreducible factor in $K[\mathbf{x}]$. Since each such $P_{i}(m, \bullet) \in K[\mathbf{x}]$ has at least one irreducible factor, by comparing (5.3) with (5.2), we see that each $e_{i}$ must be divisible by $d$, and thus

$$
f\left(x_{0}, \mathbf{x}\right)=Q\left(x_{0}\right) \mathbf{x}^{\mathbf{i}} G\left(x_{0}, \mathbf{x}\right)^{d}
$$

for some $G \in K\left[x_{0}, \mathbf{x}\right]$. Letting $\beta \in K^{*}$ be the leading coefficient of $Q$, we have following factorization

$$
Q=\beta Q_{0} Q_{1}^{d}
$$

where $Q_{0}, Q_{1} \in K\left[x_{0}\right]$ is monic such that $Q_{0}$ is $d$-th power free in $K\left[x_{0}\right]$. Choose $\gamma_{1}, \gamma_{2} \in \bar{K}$ such that

$$
\gamma_{1}^{d}=\beta \quad \text { and } \quad \gamma_{2}^{d}=\mathbf{u}^{\mathbf{i}} .
$$


By (5.1), (5.4), (5.5) and (5.6), we see that

$$
Q_{0}(m)=b(m)\left(\left(\gamma_{1} \gamma_{2}^{m}\right)^{d} Q_{1}(m)^{d} G\left(m, u_{1}^{m}, \ldots, u_{n}^{m}\right)^{d}\right)^{-1}
$$

is a $d$-th power in the function field $K\left(\gamma_{1}, \gamma_{2}\right)$ over $\mathbf{k}$ for these infinitely many $m \in \mathcal{N}$. Now Theorem 23 implies that $Q_{0} \in \mathbf{k}\left[x_{0}\right]$. Therefore, our desired conclusion holds with $R:=Q_{0}$ and $a$ given by $m \mapsto$ $\gamma_{1} \gamma_{2}^{m} Q_{1}(m) G\left(m, u_{1}^{m}, \ldots, u_{n}^{m}\right)$.

\section{Proof of the GCD Theorems}

\subsection{Key Theorems.}

We first recall some definitions in order to reformulate Theorem 2.2 in [14, which deal with the case when the coefficients of the linear forms are in $K$ instead of constants, i.e., in $\mathbf{k}$. Consider $q$ (nonzero) linear forms $L_{j}:=a_{j 0} X_{0}+\cdots+a_{j n} X_{n}, 1 \leq j \leq q$, with each $a_{j k}$ in $K$. Recall that the Weil function associated with $L_{j}$ at a place $\mathbf{p}$ of $K$ is defined by sending those $\mathbf{a} \in \mathbb{P}^{n}(K)$ with $L_{j}(\mathbf{a}) \neq 0$ to

$$
\lambda_{L_{j}, \mathbf{p}}(\mathbf{a}):=v_{\mathbf{p}}\left(L_{j}(\mathbf{a})\right)-v_{\mathbf{p}}(\mathbf{a})-v_{\mathbf{p}}\left(L_{j}\right) .
$$

For any finite-dimensional vector subspace $V \subset K$ over $\mathbf{k}$ and any positive integer $r$, we denote by $V(r)$ the vector space over $\mathbf{k}$ spanned by the set of all products of $r$ (non-necessarily distinct) elements from $V$. It is easy to show (e.g., [13, Lemma 6]) that $\operatorname{dim} V(r+1) \geq \operatorname{dim} V(r)$ for each $r$ and $\lim \inf _{r \rightarrow \infty} \operatorname{dim} V(r+1) / \operatorname{dim} V(r)=1$. Applying this inequality with $V$ replaced by $V(e)$, we see that for each $e \in \mathbb{N}$

$$
\liminf _{r \rightarrow \infty} \operatorname{dim} V(e r+e) / \operatorname{dim} V(e r)=1 .
$$

Definition 24. Let $E \subset K$ be a vector space over $\mathbf{k}$. We say that $y_{1}, \ldots, y_{m} \in K$ are linearly nondegenerate over $E$ if whenever we have a linear combination $\sum_{i=1}^{m} a_{i} y_{i}=0$ with $a_{i} \in E$, then $a_{i}=0$ for each $i$; otherwise we say that they are linearly degenerate over $E$. Similarly, a point $\mathbf{x}=\left[x_{0}: x_{1}: \cdots: x_{n}\right] \in \mathbb{P}^{n}(K)$, with each $x_{i} \in K$, is said to be linearly degenerate (resp. linearly nondegenerate) over $E$ if $x_{0}, \ldots, x_{0}$ is linearly degenerate (resp. nondegenerate) over $E$.

We obtain the following variant of Theorem 2.2 in [14 from its proof.

Theorem 25. Consider the collection $\mathcal{L}:=\left\{L_{1}, \ldots, L_{q}\right\}$ of linear forms $L_{i}=\sum_{j=0}^{n} a_{i j} X_{j} \in K\left[X_{0}, \ldots, X_{n}\right]$, $1 \leq i \leq q$, and define

$$
h(\mathcal{L}):=-\sum_{\mathbf{p} \in C(\mathbf{k})} \min _{1 \leq i \leq q, 0 \leq j \leq n} v_{\mathbf{p}}\left(a_{i j}\right) .
$$

Let $V_{\mathcal{L}} \subset K$ be the vector subspace over $\mathbf{k}$ spanned by the set consisting of all the $a_{i j}$. Suppose that $\mathbf{a} \in \mathbb{P}^{n}(K)$ is linearly nondegenerate over $V_{\mathcal{L}}(r+1)$ for some positive integer $r$, then

$$
\sum_{\mathbf{p} \in S} \max _{J} \sum_{j \in J} \lambda_{L_{j}, \mathbf{p}}(\mathbf{a}) \leq \frac{w}{u}(n+1)\left(h(\mathbf{a})+(r+2) h(\mathcal{L})+\frac{n w+w-1}{2} \max \{0,2 \mathfrak{g}-2+|S|\}\right),
$$


where the maximum is taken over all subsets $J \subset\{1, \ldots, q\}$ such that those linear forms $L_{j}$ with $j \in J$ are linearly independent over $K$, and we denote by $w:=\operatorname{dim}_{\mathbf{k}} V_{\mathcal{L}}(r+1)$ and $u:=\operatorname{dim}_{\mathbf{k}} V_{\mathcal{L}}(r)$.

We now formulate the following technical theorem of estimating the counting function of the gcd. The proof is adapted from [7] and [8] with more control on the coefficients of the constructed linear forms so that all the constants involved can be computed effectively.

Theorem 26. Let $F_{1}, F_{2} \in K\left[x_{1}, \cdots, x_{n}\right]$ be coprime polynomials of the same degree $d>0$. Assume that one of the coefficients in the expansion of $F_{i}$ is 1 for each $i \in\{1,2\}$. For every positive integer $m \geq 2 d$, we let $M:=M_{m}:=2\left(\begin{array}{c}m+n-d \\ n\end{array}\right)-\left(\begin{array}{c}m+n-2 d \\ n\end{array}\right)$ and $M^{\prime}:=M_{m}^{\prime}:=\left(\begin{array}{c}m+n \\ n\end{array}\right)-M$. For every positive integer $r$, we denote by $V_{F_{1}, F_{2}}(r)$ the (finite-dimensional) vector space over $\mathbf{k}$ spanned by $\prod_{\alpha} \alpha^{n_{\alpha}}$, where $\alpha$ runs over all non-zero coefficients of $F_{1}$ and $F_{2}, n_{\alpha} \geq 0$ and $\sum n_{\alpha}=r$; we also put $d_{r}:=\operatorname{dim}_{\mathbf{k}} V_{F_{1}, F_{2}}(r)$. Then $M_{m}^{\prime}$ has order $O\left(m^{n-2}\right)$; moreover, if for some $\mathbf{g}=\left(g_{1}, \ldots, g_{n}\right) \in\left(\mathcal{O}_{S}^{*}\right)^{n}$ those $\mathbf{g}^{\mathbf{i}}$ with $\mathbf{i} \in \mathbb{Z}_{\geq 0}^{n}$ and $|\mathbf{i}| \leq m$ are linearly nondegenerate over $V_{F_{1}, F_{2}}(M r+1)$ for some positive integer $m \geq 2 d$, then we have the following estimate

$$
\begin{aligned}
& M N_{S, \operatorname{gcd}}\left(F_{1}(\mathbf{g}), F_{2}(\mathbf{g})\right) \\
\leq & \left(M^{\prime}+\frac{d_{M r}}{d_{M(r-1)}} M-M\right) m n \max _{1 \leq i \leq n} h\left(g_{i}\right)+c M\left(h\left(F_{1}\right)+h\left(F_{2}\right)\right)+c^{\prime} M \max \{0,2 \mathfrak{g}-2+|S|\},
\end{aligned}
$$

where $c:=\frac{d_{M r}}{d_{M(r-1)}}(1+M(r+1))$ and $c^{\prime}:=\frac{d_{M r}^{2} M}{2 d_{M(r-1)}}$.

Proof. We first make some convenient settings. We denote by $\mathbf{x}:=\left(x_{1}, \ldots, x_{n}\right)$ an $n$-tuple of $n$ (algebraically independent) variables. Let $m$ be a positive integer. For a subset $T \subset K[\mathbf{x}]$, we let

$$
T_{m}=\{f \in T: \operatorname{deg} f \leq m\} .
$$

By the assumption that one of the coefficients in the expansion of $F_{i}$ is 1 for each $i \in\{1,2\}$, we note that

$$
v_{\mathbf{p}}\left(F_{i}\right) \leq 0 \quad \text { for every } i \in\{1,2\} \text { and } \mathbf{p} \in C(\mathbf{k}) .
$$

Consider the ideal $\left(F_{1}, F_{2}\right) \subset K[\mathbf{x}]$. If $\left(F_{1}, F_{2}\right)=(1)=K[\mathbf{x}]$, then it is elementary to show that $N_{S \text {,gcd }}\left(F_{1}(\mathbf{g}), F_{2}(\mathbf{g})\right)$ is bounded by some constant independent of $\mathbf{g}$. Therefore, we assume that the ideal $\left(F_{1}, F_{2}\right)$ is proper. For $\mathbf{i}=\left(i_{1}, \cdots, i_{n}\right) \in \mathbb{Z}_{\geq 0}^{n}$, we let $\mathbf{x}^{\mathbf{i}}:=x_{1}^{i_{1}} \cdots x_{n}^{i_{n}}$, and $\mathbf{g}^{\mathbf{i}}:=g_{1}^{i_{1}} \cdots g_{n}^{i_{n}}$. By Lemma 2.11 of $\left[8\right.$, we note that $M=\operatorname{dim}_{K}\left(F_{1}, F_{2}\right)_{m}$, and may choose a basis $\left\{\phi_{1}, \ldots, \phi_{M}\right\}$ of the $K$-vector space $\left(F_{1}, F_{2}\right)_{m}$ such that each $\phi_{j}$ is of the form $\mathbf{x}^{\mathbf{i}} F_{j}$ with $|\mathbf{i}|:=i_{1}+\cdots+i_{n} \leq m-d$ and $j \in\{1,2\}$. Put

$$
\Phi:=\left(\phi_{1}, \ldots, \phi_{M}\right) \quad \text { and } \quad \Phi(\mathrm{g}):=\left(\phi_{1}(\mathrm{~g}), \ldots, \phi_{M}(\mathrm{~g})\right) .
$$

For each $\mathbf{p} \in S$, we construct a subset $B_{\mathbf{p}} \subset K[\mathbf{x}]_{m}$, consisting of only monomials, whose images in the $K$-linear space $V_{m}:=K[\mathbf{x}]_{m} /\left(F_{1}, F_{2}\right)_{m}$ form one of its bases as follows. Choose a monomial $\mathbf{x}^{\mathbf{i}_{\mathbf{p}, 1}} \in$ $K\left[x_{1}, \ldots, x_{n}\right]_{m}$ so that $v_{\mathbf{p}}\left(\mathbf{g}^{\mathbf{i}_{\mathbf{p}, 1}}\right)$ is maximum subject to the condition $\mathbf{x}^{\mathbf{i}_{\mathbf{p}, 1}} \notin\left(F_{1}, F_{2}\right)$. If $\mathbf{x}^{\mathbf{i}_{\mathbf{p}, 1}}, \ldots, \mathbf{x}^{\mathbf{i}_{\mathbf{p}, j}}$ have been constructed such that their images in $V_{m}$ are $K$-linearly independent but don't span the whole 
$V_{m}$, then we let $\mathbf{x}^{\mathbf{i}_{\mathbf{p}, j+1}} \in K\left[x_{1}, \ldots, x_{n}\right]_{m}$ be a monomial such that $v_{\mathbf{p}}\left(\mathbf{g}^{\mathbf{i}_{\mathbf{p}, j+1}}\right)$ is maximum subject to the condition that the images of $\mathbf{x}^{\mathbf{i}_{\mathbf{p}}, 1}, \ldots, \mathbf{x}^{\mathbf{i}_{\mathbf{p}, j+1}}$ in $V_{m}$ are $K$-linearly independent; otherwise we stop. Because $\operatorname{dim}_{K} V_{m}=\operatorname{dim}_{K} K[\mathbf{x}]_{m}-\operatorname{dim}_{K}\left(F_{1}, F_{2}\right)_{m}=\left(\begin{array}{c}m+n \\ n\end{array}\right)-M=M^{\prime}$, we will eventually stop and obtain that $B_{\mathbf{p}}:=\left\{\mathbf{x}^{\mathbf{i}_{\mathbf{p}, 1}}, \ldots, \mathbf{x}^{\mathbf{i}_{\mathbf{p}, M^{\prime}}}\right\} \subset K[\mathbf{x}]_{m}$ is a set of monomials whose images in $V_{m}$ form one of its $K$-linear bases such that

$$
v_{\mathbf{p}}\left(\mathbf{g}^{\mathbf{i}_{\mathbf{p}, 1}}\right) \geq v_{\mathbf{p}}\left(\mathbf{g}^{\mathbf{i}_{\mathbf{p}, 2}}\right) \geq \cdots \geq v_{\mathbf{p}}\left(\mathbf{g}^{\mathbf{i}_{\mathbf{p}, M^{\prime}}}\right) \geq v_{\mathbf{p}}\left(\mathbf{g}^{\mathbf{i}_{\mathbf{p}}(i)}\right)
$$

for each $i \in\{1, \ldots, M\}$, where we denote by $\left\{\mathbf{i}_{\mathbf{p}}(1), \ldots, \mathbf{i}_{\mathbf{p}}(M)\right\}$ the set of those $\mathbf{i} \in \mathbb{Z}_{\geq 0}^{n}$ with $|\mathbf{i}| \leq m$ and $\mathbf{i} \notin I_{\mathbf{p}}$, where

$$
I_{\mathbf{p}}:=\left\{\mathbf{i}_{\mathbf{p}, 1}, \ldots, \mathbf{i}_{\mathbf{p}, M^{\prime}}\right\} .
$$

By direction calculation, we find that $M_{m}^{\prime}=\left(\begin{array}{c}m+n \\ n\end{array}\right)-2\left(\begin{array}{c}m+n-d \\ n\end{array}\right)+\left(\begin{array}{c}m+n-2 d \\ n\end{array}\right)=O\left(m^{n-2}\right)$. Alternatively, since $F_{1}$ and $F_{2}$ are coprime, the ideal $(F, G)$ defines a closed subset of $\mathbb{P}^{n}$ of codimension at least 2 , and it follows from the theory of Hilbert functions and Hilbert polynomials that $M_{m}^{\prime}=\operatorname{dim}_{K} V_{m}=O\left(m^{n-2}\right)$.

For each $i \in\{1, \ldots, M\}$, we have

$$
\mathbf{x}^{\mathbf{i}_{\mathbf{p}}(i)}+\sum_{j=1}^{M^{\prime}} c_{\mathbf{p}, i, j} \mathbf{x}^{\mathbf{i}_{\mathbf{p}, j}} \in\left(F_{1}, F_{2}\right)_{m}
$$

for some (unique) choice of coefficients $c_{\mathbf{p}, i, j} \in K$; by expressing $\mathbf{x}^{\mathbf{i}_{\mathbf{p}}(i)}+\sum_{j=1}^{M^{\prime}} c_{\mathbf{p}, i, j} \mathbf{x}^{\mathbf{i}_{\mathbf{p} j}}$ as a (unique) $K$-linear combination of $\phi_{1}, \ldots, \phi_{M}$, we let

$$
L_{\mathbf{p}, i}:=\sum_{\ell=1}^{M} b_{\mathbf{p}, i, \ell} y_{\ell} \in K\left[y_{1}, \ldots, y_{M}\right]
$$

be a linear form over $K$ such that

$$
L_{\mathbf{p}, i}(\Phi(\mathbf{x}))=c_{\mathbf{p}}\left(\mathbf{x}^{\mathbf{i}_{\mathbf{p}}(i)}+\sum_{j=1}^{M^{\prime}} c_{\mathbf{p}, i, j} \mathbf{x}^{\mathbf{i}_{\mathbf{p}, j}}\right),
$$

where $c_{\mathbf{p}} \in K^{*}$ will be chosen later.

By the choice of the $\phi_{\ell}$, we may write

$$
\phi_{\ell}=\sum_{s=1}^{M} \alpha_{\mathbf{p}, \ell, s} \mathbf{x}^{\mathbf{i}_{\mathbf{p}}(s)}+\sum_{j=1}^{M^{\prime}} \alpha_{\mathbf{p}, \ell, \mathbf{i}_{\mathbf{p}, j}} \mathbf{x}^{\mathbf{i}_{\mathbf{p}, j}}
$$

where both $\alpha_{\mathbf{p}, \ell, i}$ and $\alpha_{\mathbf{p}, \ell, \mathbf{i}_{\mathbf{p}, j}}$ are coefficients of either $F_{1}$ or $F_{2}$, thus

$$
\min \left\{v_{\mathbf{p}}\left(\alpha_{\mathbf{p}, \ell, \mathbf{i}_{\mathbf{p}, j}}\right), v_{\mathbf{p}}\left(\alpha_{\mathbf{p}, \ell, i}\right)\right\} \geq v_{\mathbf{p}}\left(F_{1}\right)+v_{\mathbf{p}}\left(F_{2}\right) \quad \text { for each } \ell, i, j .
$$

Combining (6.7) and (6.9), we have

$$
L_{\mathbf{p}, i}(\Phi(\mathbf{x}))=\sum_{\ell=1}^{M} b_{\mathbf{p}, i, \ell}\left(\sum_{s=1}^{M} \alpha_{\mathbf{p}, \ell, s} \mathbf{x}^{\mathbf{i}_{\mathbf{p}}(s)}+\sum_{j=1}^{M^{\prime}} \alpha_{\mathbf{p}, \ell, \mathbf{i}_{\mathbf{p}, j}} \mathbf{x}^{\mathbf{i}_{\mathbf{p}, j}}\right) .
$$

Note that if we take $c_{\mathbf{p}}=1$, then by comparing (6.8) with (6.11), we find that

$$
\operatorname{det}\left(b_{\mathbf{p}, i, \ell}\right)_{1 \leq \ell, i \leq M} \operatorname{det}\left(\alpha_{\mathbf{p}, \ell, s}\right)_{1 \leq \ell, s \leq M}=1 .
$$


From now on, we let

$$
c_{\mathbf{p}}:=\operatorname{det}\left(\alpha_{\mathbf{p}, \ell, s}\right)_{1 \leq \ell, s \leq M} \neq 0
$$

and note that $c_{\mathbf{p}} \in V_{F_{1}, F_{2}}(M)$. With this choice of $c_{\mathbf{p}}$, we compare (6.8) with (6.11) again and see that the inverse of $\left(\alpha_{\mathbf{p}, \ell, s}\right)_{1 \leq \ell, s \leq M}$ is $c_{\mathbf{p}}^{-1}\left(b_{\mathbf{p}, i, \ell}\right)_{1 \leq \ell, i \leq M}$, which shows that

$$
b_{\mathbf{p}, i, \ell} \in V_{F_{1}, F_{2}}(M-1)
$$

for each $i, \ell$ by Cramer's rule. This comparison also gives

$$
\begin{aligned}
c_{\mathbf{p}} & =\sum_{\ell=1}^{M} b_{\mathbf{p}, i, \ell} \alpha_{\mathbf{p}, \ell, i} \quad \text { for each } 1 \leq i \leq M, \\
c_{\mathbf{p}} c_{\mathbf{p}, i, j} & =\sum_{\ell=1}^{M} b_{\mathbf{p}, i, \ell} \alpha_{\mathbf{p}, \ell, \mathbf{i}_{\mathbf{p}, j}} \quad \text { for each } 1 \leq i \leq M \text { and } 1 \leq j \leq M^{\prime} .
\end{aligned}
$$

From (6.5), 6.8), 6.14), 6.15), 6.10) and (6.4), we have

$$
\begin{aligned}
v_{\mathbf{p}}\left(L_{\mathbf{p}, i}(\Phi(\mathbf{g}))\right) & \geq v_{\mathbf{p}}\left(\mathbf{g}^{\mathbf{i}_{\mathbf{p}}(i)}\right)+\min _{j}\left\{v_{\mathbf{p}}\left(c_{\mathbf{p}}\right), v_{\mathbf{p}}\left(c_{\mathbf{p}} c_{\mathbf{p}, i, j}\right)\right\} \\
& \geq v_{\mathbf{p}}\left(\mathbf{g}^{\mathbf{i}_{\mathbf{p}}(i)}\right)+\min _{j}\left\{\min _{\ell} v_{\mathbf{p}}\left(b_{\mathbf{p}, i, \ell}\right)+\min _{\ell} v_{\mathbf{p}}\left(\alpha_{\mathbf{p}, \ell, i}\right), \min _{\ell} v_{\mathbf{p}}\left(b_{\mathbf{p}, i, \ell}\right)+\min _{\ell} v_{\mathbf{p}}\left(\alpha_{\mathbf{p}, \ell, \mathbf{i}_{\mathbf{p}, j}}\right)\right\} \\
& \geq v_{\mathbf{p}}\left(\mathbf{g}^{\mathbf{i}_{\mathbf{p}}(i)}\right)+\min _{\ell} v_{\mathbf{p}}\left(b_{\mathbf{p}, i, \ell}\right)+v_{\mathbf{p}}\left(F_{1}\right)+v_{\mathbf{p}}\left(F_{2}\right)
\end{aligned}
$$

which gives the following key inequality

$$
v_{\mathbf{p}}\left(L_{\mathbf{p}, i}(\Phi(\mathbf{g}))\right)-v_{\mathbf{p}}\left(L_{\mathbf{p}, i}\right) \geq v_{\mathbf{p}}\left(\mathbf{g}^{\mathbf{i}_{\mathbf{p}}(i)}\right)+v_{\mathbf{p}}\left(F_{1}\right)+v_{\mathbf{p}}\left(F_{2}\right) .
$$

Thus, by the construction of (6.6) and the fact that $\mathbf{g}^{\mathbf{i}} \in \mathcal{O}_{S}^{*}$ for each $\mathbf{i}$, we have

$$
\begin{aligned}
\sum_{\mathbf{p} \in S} \sum_{1 \leq i \leq M} v_{\mathbf{p}}\left(\mathbf{g}^{\mathbf{i}_{\mathbf{p}}(i)}\right) & =\sum_{\mathbf{p} \in S} \sum_{|\mathbf{i}| \leq m} v_{\mathbf{p}}\left(\mathbf{g}^{\mathbf{i}}\right)-\sum_{\mathbf{p} \in S} \sum_{|\mathbf{i}| \leq m, \mathbf{i} \in I_{\mathbf{p}}} v_{\mathbf{p}}\left(\mathbf{g}^{\mathbf{i}}\right) \\
& \geq \sum_{|\mathbf{i}| \leq m} \sum_{\mathbf{p} \in S} v_{\mathbf{p}}\left(\mathbf{g}^{\mathbf{i}}\right)-\left|I_{\mathbf{p}}\right| m \sum_{\mathbf{p} \in S} \sum_{j=1}^{n} v_{\mathbf{p}}^{0}\left(g_{j}\right) \\
& =-M^{\prime} m \sum_{j=1}^{n} h\left(g_{j}\right) \\
& \geq-M^{\prime} m n \max _{1 \leq j \leq n} h\left(g_{j}\right) .
\end{aligned}
$$

By the choice of these $\phi_{i} \in K\left[x_{1}, \ldots, x_{n}\right]_{m}$, together with (6.4), we have

$$
\begin{aligned}
v_{\mathbf{p}}\left(\phi_{i}(\mathbf{g})\right) & \geq m \min \left\{v_{\mathbf{p}}(\mathbf{g}), 0\right\}+v_{\mathbf{p}}\left(F_{1}\right)+v_{\mathbf{p}}\left(F_{2}\right) \\
& \geq-m \sum_{j=1}^{n} v_{\mathbf{p}}^{\infty}\left(g_{j}\right)+v_{\mathbf{p}}\left(F_{1}\right)+v_{\mathbf{p}}\left(F_{2}\right)
\end{aligned}
$$

for every $\mathbf{p} \in C(\mathbf{k})$. It follows that 


$$
h(\Phi(\mathbf{g})) \leq m n \max _{1 \leq i \leq n} h\left(g_{i}\right)+h\left(F_{1}\right)+h\left(F_{2}\right) .
$$

Also, with the fact that $\mathbf{g}^{\mathbf{i}} \in \mathcal{O}_{S}^{*}$ for each $\mathbf{i}$, we note for every $\mathbf{p} \notin S$ that $v_{\mathbf{p}}\left(\phi_{i}(\mathbf{g})\right)=v_{\mathbf{p}}\left(F_{\epsilon_{i}}(\mathbf{g})\right) \geq v_{\mathbf{p}}\left(F_{\epsilon_{i}}\right)$ with some $\epsilon_{i} \in\{1,2\}$, and hence, together with (6.4), we have that

$$
\begin{aligned}
v_{\mathbf{p}}\left(\phi_{i}(\mathbf{g})\right) & \geq \min \left\{v_{\mathbf{p}}\left(F_{1}(\mathbf{g})\right), v_{\mathbf{p}}\left(F_{2}(\mathbf{g})\right)\right\} \\
& \geq \min \left\{v_{\mathbf{p}}^{0}\left(F_{1}(\mathbf{g})\right), v_{\mathbf{p}}^{0}\left(F_{2}(\mathbf{g})\right)\right\}+v_{\mathbf{p}}\left(F_{1}\right)+v_{\mathbf{p}}\left(F_{2}\right) .
\end{aligned}
$$

By (6.20), we have

$$
\begin{aligned}
N_{S, \text { gcd }}\left(F_{1}(\mathbf{g}), F_{2}(\mathbf{g})\right) & =\sum_{\mathbf{p} \notin S} \min \left\{v_{\mathbf{p}}^{0}\left(F_{1}(\mathbf{g})\right), v_{\mathbf{p}}^{0}\left(F_{2}(\mathbf{g})\right)\right\} \\
& \leq \sum_{\mathbf{p} \notin S} \min _{i} v_{\mathbf{p}}\left(\phi_{i}(\mathbf{g})\right)+\sum_{\mathbf{p} \notin S}-\left(v_{\mathbf{p}}\left(F_{1}\right)+v_{\mathbf{p}}\left(F_{2}\right)\right) \\
& =\sum_{\mathbf{p} \notin S} v_{\mathbf{p}}(\Phi(\mathbf{g}))+\sum_{\mathbf{p} \notin S}-\left(v_{\mathbf{p}}\left(F_{1}\right)+v_{\mathbf{p}}\left(F_{2}\right)\right) \\
& =-h(\Phi(\mathbf{g}))-\sum_{\mathbf{p} \in S} v_{\mathbf{p}}(\Phi(\mathbf{g}))+\sum_{\mathbf{p} \notin S}-\left(v_{\mathbf{p}}\left(F_{1}\right)+v_{\mathbf{p}}\left(F_{2}\right)\right) .
\end{aligned}
$$

By (6.13), we may choose a finite collection $\mathcal{L}$ of linear forms over $K$ such that $L_{\mathbf{p}, i} \in \mathcal{L}$ for each $\mathbf{p} \in S$ and $i \in\{1, \ldots M\}$, that the finite-dimensional $\mathbf{k}$-linear subspace $V:=V_{F_{1}, F_{2}}(M)$ is spanned by the set of all coefficients of linear forms in $\mathcal{L}$, and that

$$
h(\mathcal{L}) \leq M\left(h\left(F_{1}\right)+h\left(F_{2}\right)\right) .
$$

Since those $\mathbf{g}^{\mathbf{i}}$ with $\mathbf{i} \in \mathbb{Z}_{\geq 0}^{n}$ and $|\mathbf{i}| \leq m$ are linearly nondegenerate over $V_{F_{1}, F_{2}}(M r+1)$, we must have that $\Phi(\mathrm{g}) \in \mathbb{P}^{M-1}(K)$ is linearly nondegenerate over $V_{F_{1}, F_{2}}(M r)=V(r)$. By (6.12), we note that elements from $\left\{L_{\mathbf{p}, i}(\Phi(\mathbf{x})) \mid 1 \leq i \leq M\right\}$ are linearly independent over $K$; thus the linear forms $L_{\mathbf{p}, i}, 1 \leq i \leq M$, are linearly independent over $K$. Noting that $d_{M r}=\operatorname{dim}_{\mathbf{k}} V(r)$ and $d_{M(r-1)}:=\operatorname{dim}_{\mathbf{k}} V(r-1)$, we obtain from Theorem 25] and (6.22) that

$\sum_{\mathbf{p} \in S} \sum_{1 \leq i \leq M} \lambda_{L_{\mathbf{p}, i}, \mathbf{p}}(\Phi(\mathbf{g})) \leq \frac{d_{M r} M}{d_{M(r-1)}}\left(h(\Phi(\mathbf{g}))+(r+1) M\left(h\left(F_{1}\right)+h\left(F_{2}\right)\right)+\frac{M d_{M r}-1}{2} \max \{0,2 \mathfrak{g}-2+|S|\}\right)$.

Together with (6.17), 6.18) and (6.21), we have

$$
\begin{aligned}
\sum_{\mathbf{p} \in S} \sum_{1 \leq i \leq M} \lambda_{L_{\mathbf{p}, i}, \mathbf{p}}(\Phi(\mathbf{g}))= & \sum_{\mathbf{p} \in S} \sum_{1 \leq i \leq M}\left(v_{\mathbf{p}}\left(L_{\mathbf{p}, i}(\Phi(\mathbf{g}))\right)-v_{\mathbf{p}}\left(L_{\mathbf{p}, i}\right)\right)-M \sum_{\mathbf{p} \in S} v_{\mathbf{p}}(\Phi(\mathbf{g})) \\
\geq & \sum_{\mathbf{p} \in S} \sum_{1 \leq i \leq M} v_{\mathbf{p}}\left(\mathbf{g}^{\mathbf{i}_{\mathbf{p}}(i)}\right)+M \sum_{\mathbf{p} \in S}\left(v_{\mathbf{p}}\left(F_{1}\right)+v_{\mathbf{p}}\left(F_{2}\right)\right)+M N_{S, \operatorname{gcd}}\left(F_{1}(\mathbf{g}), F_{2}(\mathbf{g})\right) \\
& \quad+M h(\Phi(\mathbf{g}))+M \sum_{\mathbf{p} \notin S}\left(v_{\mathbf{p}}\left(F_{1}\right)+v_{\mathbf{p}}\left(F_{2}\right)\right) \\
\geq & -M^{\prime} m n \max _{1 \leq j \leq n} h\left(g_{j}\right)+M N_{S, \operatorname{gcd}}\left(F_{1}(\mathbf{g}), F_{2}(\mathbf{g})\right)+M\left(h(\Phi(\mathbf{g}))-h\left(F_{1}\right)-h\left(F_{2}\right)\right) .
\end{aligned}
$$


Combining (6.24) with (6.23) and (6.19), we get

$$
\begin{aligned}
& M N_{S, \operatorname{gcd}}\left(F_{1}(\mathbf{g}), F_{2}(\mathbf{g})\right) \\
\leq & M^{\prime} m n \max _{1 \leq j \leq n} h\left(g_{j}\right)+M\left(\frac{d_{M r}}{d_{M(r-1)}}-1\right) h(\Phi(\mathbf{g}))+\left(\frac{d_{M r}}{d_{M(r-1)}} M^{2}(r+1)+M\right)\left(h\left(F_{1}\right)+h\left(F_{2}\right)\right) \\
& +\frac{d_{M r} M\left(M d_{M r}-1\right)}{2 d_{M(r-1)}} \max \{0,2 \mathfrak{g}-2+|S|\} \\
\leq & \left(M^{\prime}+\frac{d_{M r}}{d_{M(r-1)}} M-M\right) m n \max _{1 \leq i \leq n} h\left(g_{i}\right)+\left(\frac{d_{M r}}{d_{M(r-1)}}(1+M(r+1))\right) M\left(h\left(F_{1}\right)+h\left(F_{2}\right)\right) \\
& +\frac{d_{M r}^{2} M^{2}}{2 d_{M(r-1)}} \max \{0,2 \mathfrak{g}-2+|S|\} .
\end{aligned}
$$

Theorem 27. Let $F \in K\left[x_{1}, \cdots, x_{n}\right]$ be a polynomials of degree $d>0$ that does not vanish at $(0, \ldots, 0)$. Assume that one of the coefficients of $F$ is 1 . For each $r \in \mathbb{N}$, denote by $V_{F}(r)$ the (finite-dimensional) vector space over $\mathbf{k}$ spanned by $\prod_{\alpha} \alpha^{n_{\alpha}}$, where $\alpha$ runs over all (non-zero) coefficients of $F$ with $n_{\alpha} \geq 0$ and $\sum n_{\alpha}=r ;$ put $d_{r}:=\operatorname{dim}_{\mathbf{k}} V_{F}(r)$. Put $N:=\left(\begin{array}{c}n+d \\ n\end{array}\right)-1$. Let $\mathbf{g}=\left(g_{1}, \ldots, g_{n}\right) \in\left(\mathcal{O}_{S}^{*}\right)^{n}$. Suppose those $\mathbf{g}^{\mathbf{i}}$ with $\mathbf{i} \in \mathbb{Z}_{\geq 0}^{n}$ and $|\mathbf{i}| \leq d$ are linearly nondegenerate over $V_{F}(r)$. Then we have the following estimate

$$
\begin{aligned}
\sum_{\mathbf{p} \in S} v_{\mathbf{p}}^{0}(F(\mathbf{g})) \leq & \left(\frac{d_{r}}{d_{r-1}}-1\right)(N+1) d n \max _{1 \leq i \leq n} h\left(g_{i}\right)+\frac{d_{r}(N+1)}{d_{r-1}}(r+1) h(F) \\
& +\frac{d_{r}(N+1)\left(N d_{r}+d_{r}-1\right)}{2 d_{r-1}} \max \{0,2 \mathfrak{g}-2+|S|\} .
\end{aligned}
$$

Proof. Let $\Phi=\left(\phi_{0}, \phi_{1}, \ldots, \phi_{N}\right): \mathbb{P}^{n} \rightarrow \mathbb{P}^{N}$ be the $d$-tuple embedding of $\mathbb{P}^{n}$ given by the set of monomials of degree $d$ in $K\left[x_{0}, \ldots, x_{n}\right]$, where $\phi_{0}:=x_{0}^{d}$. Let $\tilde{F} \in K\left[x_{0}, \ldots, x_{n}\right]$ be the homogenization of $F$. Denote by $\tilde{\mathbf{g}}:=\left(g_{0}, g_{1}, \ldots, g_{n}\right)$, where $g_{0}:=1$.

Since each $\phi_{i}$ is a degree- $d$ monomial in $K\left[x_{0}, \ldots, x_{n}\right]$, we have $v_{\mathbf{p}}\left(\phi_{i}(\tilde{\mathbf{g}})\right) \geq d v_{\mathbf{p}}(\tilde{\mathbf{g}})=d \min \left\{v_{\mathbf{p}}(\mathbf{g}), 0\right\} \geq$ $-d \sum_{j=1}^{n} v_{\mathbf{p}}^{\infty}\left(g_{j}\right)$ for every $\mathbf{p} \in C(\mathbf{k})$ and $i \in\{0, \ldots, N\}$; thus we have

$$
h(\Phi(\tilde{\mathbf{g}})) \leq d n \max _{1 \leq i \leq n} h\left(g_{i}\right) .
$$

Also, since $\phi_{i}(\tilde{\mathbf{g}}) \in \mathcal{O}_{S}^{*}$, we have that

$$
\sum_{\mathbf{p} \in S} v_{\mathbf{p}}\left(\phi_{i}(\tilde{\mathbf{g}})\right)=0
$$

For each $i \in\{0, \ldots, N\}$, denote by $L_{i}$ the linear form corresponding to the coordinate hyperplanes $\mathbb{P}^{N}$. We also denote by $L_{\tilde{F}} \in K\left[y_{0}, \ldots, y_{N}\right]$ the linear form coming from the monomial expansion (of degree $d$ ) of $\tilde{F}$; thus $L_{\tilde{F}}(\Phi(\tilde{\mathbf{g}}))=\tilde{F}(\tilde{\mathbf{g}})$. Let $\mathcal{L}:=\left\{L_{i} \mid i \in\{0, \ldots, N\}\right\} \cup\left\{L_{\tilde{F}}\right\}$. By construction and our assumption that one of the coefficients of $F$ is 1 , we have that $h(\mathcal{L})=\tilde{h}(F)=h(F)$, that $V_{F}(1)$ is spanned by the set of all coefficients of linear forms in $\mathcal{L}$, and that

$$
v_{\mathbf{p}}(L) \leq 0 \quad \text { for every } L \in \mathcal{L} \text { and } \mathbf{p} \in C(\mathbf{k}) .
$$


We also note that any $N+1$ linear forms in $\mathcal{L}$ are linearly independent over $K$ since $F(0, \ldots, 0) \neq 0$.

For those $\mathbf{p} \in S$ and $i \in\{0, \ldots, N\}$ satisfying either $i \neq 0$ or $v_{\mathbf{p}}(F(\mathbf{g})) \leq 0$, we define $L_{\mathbf{p}, i}:=L_{i}$; for the remaining case, we define $L_{\mathbf{p}, i}:=L_{\tilde{F}}$. Hence we see

$$
\begin{array}{ll}
L_{\mathbf{p}, i}(\Phi(\tilde{\mathbf{g}}))=F(\mathbf{g}) & \text { if } i=0 \text { and } v_{\mathbf{p}}(F(\mathbf{g}))>0 ; \\
L_{\mathbf{p}, i}(\Phi(\tilde{\mathbf{g}}))=\phi_{i}(\tilde{\mathbf{g}}) \quad \text { otherwise. }
\end{array}
$$

By assumption, those $\mathbf{g}^{\mathbf{i}}$ with $\mathbf{i} \in \mathbb{Z}_{\geq 0}^{n}$ and $|\mathbf{i}| \leq d$ are linearly nondegenerate over $V_{F}(r)$, thus $\Phi(\tilde{\mathbf{g}}) \in$ $\mathbb{P}^{N}(K)$ is linearly nondegenerate over $V_{F}(r)$. Applying Theorem 25 with $\mathbf{a}=\Phi(\tilde{\mathbf{g}})$ and $V_{\mathcal{L}}=V_{F}(1)$, we have

$$
\begin{aligned}
& \sum_{\mathbf{p} \in S} \sum_{i=0}^{N}\left(v_{\mathbf{p}}\left(L_{\mathbf{p}, i}(\Phi(\tilde{\mathbf{g}}))-v_{\mathbf{p}}(\Phi(\tilde{\mathbf{g}}))-v_{\mathbf{p}}\left(L_{\mathbf{p}, i}\right)\right)\right. \\
\leq & \frac{d_{r}(N+1)}{d_{r-1}}\left(h(\Phi(\tilde{\mathbf{g}}))+(r+1) h(F)+\frac{N d_{r}+d_{r}-1}{2} \max \{0,2 \mathfrak{g}-2+|S|\}\right) .
\end{aligned}
$$

Together with (6.26), (6.28), (6.29) and (6.27), we have the following estimate for the left hand side of (6.30)

$$
\sum_{i=0}^{N} \sum_{\mathbf{p} \in S}\left(v_{\mathbf{p}}\left(L_{\mathbf{p}, i}(\Phi(\tilde{\mathbf{g}}))-v_{\mathbf{p}}(\Phi(\tilde{\mathbf{g}}))-v_{\mathbf{p}}\left(L_{\mathbf{p}, i}\right)\right) \geq \sum_{\mathbf{p} \in S}\left(v_{\mathbf{p}}^{0}(F(\mathbf{g}))-(N+1) v_{\mathbf{p}}(\Phi(\tilde{\mathbf{g}})) .\right.\right.
$$

Therefore, we can derive from (6.30) and (6.25) that

$$
\begin{aligned}
\sum_{\mathbf{p} \in S} v_{\mathbf{p}}^{0}(F(\mathbf{g})) \leq & \left(\frac{d_{r}}{d_{r-1}}-1\right)(N+1) d n \max _{1 \leq i \leq n} h\left(g_{i}\right)+\frac{d_{r}(N+1)}{d_{r-1}}(r+1) h(F) \\
& +\frac{d_{r}(N+1)\left(N d_{r}+d_{r}-1\right)}{2 d_{r-1}} \max \{0,2 \mathfrak{g}-2+|S|\}
\end{aligned}
$$

\subsection{Proof of Theorem 8 ,}

Proof of Theorem 8 . Let $\alpha$ and $\beta$ be one of the nonzero coefficients of $F$ and $G$ respectively, and put $\mathbf{g}:=$ $\left(g_{1}, \ldots, g_{n}\right) \in\left(\mathcal{O}_{S}^{*}\right)^{n}, 1 \leq j \leq n$. Since $v_{\mathbf{p}}^{0}(F(\mathbf{g})) \leq v_{\mathbf{p}}^{0}\left(\frac{1}{\alpha} F(\mathbf{g})\right)+v_{\mathbf{p}}^{0}(\alpha)$ and $v_{\mathbf{p}}^{0}(G(\mathbf{g})) \leq v_{\mathbf{p}}^{0}\left(\frac{1}{\beta} G(\mathbf{g})\right)+v_{\mathbf{p}}^{0}(\beta)$, we have

$$
N_{S, \operatorname{gcd}}(F(\mathbf{g}), G(\mathbf{g})) \leq N_{S, \operatorname{gcd}}\left(\frac{1}{\alpha} F(\mathbf{g}), \frac{1}{\beta} G(\mathbf{g})\right)+\tilde{h}(F)+\tilde{h}(G)
$$

and

$$
h_{\mathrm{gcd}}(F(\mathbf{g}), G(\mathbf{g})) \leq h_{\mathrm{gcd}}\left(\frac{1}{\alpha} F(\mathbf{g}), \frac{1}{\beta} G(\mathbf{g})\right)+\tilde{h}(F)+\tilde{h}(G) .
$$

Then by elementary reductions, which we omit, it suffices to prove the theorem for $\frac{1}{\alpha} F$ and $\frac{1}{\beta} G$. Therefore, we assume that, with respect to some fixed total ordering on the set of monomials in $K\left[x_{1}, \ldots, x_{n}\right]$, the coefficient attached to the largest monomial appearing in $F$ (resp. in $G$ ) is 1. In this case, $\tilde{h}\left(F^{e}\right)=h\left(F^{e}\right)=e h(F)=e \tilde{h}(F)$ for every $e \in \mathbb{N}$, thus we may assume that $F$ and $G$ have the same degree $d$ via replacing $F$ (resp. $G$ ) by some of its powers. 
Let $\epsilon>0$ be given. We first choose $m$ sufficiently large so that $m \geq 2 d$ and

$$
\frac{M^{\prime} m n}{M} \leq \frac{\epsilon}{4}
$$

where $M:=M_{m}:=2\left(\begin{array}{c}m+n-d \\ n\end{array}\right)-\left(\begin{array}{c}m+n-2 d \\ n\end{array}\right)$ and $M^{\prime}:=M_{m}^{\prime}:=\left(\begin{array}{c}m+n \\ n\end{array}\right)-M$; this is possible because $M_{m}=$ $\frac{m^{n}}{n !}+O\left(m^{n-1}\right)$ and $M^{\prime}=O\left(m^{n-2}\right)$ (by the proof of Theorem 26). By (6.1) we may then choose a sufficiently large integer $r \in \mathbb{N}$ such that

$$
\frac{w}{u}-1 \leq \frac{\epsilon}{4 m n}
$$

where $w:=\operatorname{dim}_{\mathbf{k}} V_{F, G}(M r)$ and $u:=\operatorname{dim}_{\mathbf{k}} V_{F, G}(M r-M)$ (as in Theorem 26); in the case where $F(0, \ldots, 0) \neq 0$, we further require that

$$
\frac{w^{\prime}}{u^{\prime}}-1 \leq \frac{\epsilon}{8 d n(N+1)}
$$

where $w^{\prime}:=\operatorname{dim} V_{F}(r), u^{\prime}:=\operatorname{dim} V_{F}(r-1)$ and $N:=\left(\begin{array}{c}n+d \\ n\end{array}\right)-1$ (as in Theorem 27).

We first consider when those $\mathbf{g}^{\mathbf{i}}$ with $\mathbf{i} \in \mathbb{Z}_{\geq 0}^{n}$ and $|\mathbf{i}| \leq m$ are linearly degenerate over $V_{F, G}(M r+1)$, i.e. there is a non-trivial relation

$$
\sum_{\mathbf{i}} \alpha_{\mathbf{i}} \mathbf{g}^{\mathbf{i}}=0
$$

where the sum runs over those $\mathbf{i} \in \mathbb{Z}_{\geq 0}^{n}$ and $|\mathbf{i}| \leq m$, and $\alpha_{\mathbf{i}} \in V_{F, G}(M r+1)$ for each $\mathbf{i}$ such that $\alpha_{\mathbf{i}_{0}} \neq 0$ for some $\mathbf{i}_{0}$. Then we have

$$
\sum_{\mathbf{i} \neq \mathbf{i}_{0}} \frac{\alpha_{\mathbf{i}}}{\alpha_{\mathbf{i}_{0}}} \mathbf{g}^{\mathbf{i}-\mathbf{i}_{0}}=-1
$$

Since $\mathbf{g} \in\left(\mathcal{O}_{S}^{*}\right)^{n}$ and the number of zeros and poles of each $\alpha_{\mathbf{i}}$ appearing in (6.34) is bounded by $2 h\left(\alpha_{\mathbf{i}}\right) \leq$ $2(M r+1)(\tilde{h}(F)+\tilde{h}(G))$, we can apply Theorem 14 with some $S^{\prime}$ including $S$ and the zeros and poles of those $\alpha_{\mathbf{i}}$ appearing in (6.34) to get

$$
h\left(\frac{\alpha_{\mathbf{i}}}{\alpha_{\mathbf{i}_{0}}} \mathbf{g}^{\mathbf{i}-\mathbf{i}_{0}}\right) \leq \tilde{c} \max \left\{0,2 \mathfrak{g}-2+|S|+2(M r+1)\left(\begin{array}{c}
n+m \\
n
\end{array}\right)(\tilde{h}(F)+\tilde{h}(G))\right\},
$$

where $\tilde{c}:=\frac{1}{2}\left(\left(\begin{array}{c}n+m \\ n\end{array}\right)-1\right)\left(\left(\begin{array}{c}n+m \\ n\end{array}\right)-2\right)$. Then

$$
\begin{aligned}
h\left(\mathbf{g}^{\mathbf{i}-\mathbf{i}_{0}}\right) & \leq h\left(\frac{\alpha_{\mathbf{i}}}{\alpha_{\mathbf{i}_{0}}}\right)+h\left(\frac{\alpha_{\mathbf{i}}}{\alpha_{\mathbf{i}_{0}}} \mathbf{g}^{\mathbf{i}-\mathbf{i}_{0}}\right) \\
& \leq 2(M r+1)\left(\tilde{c}\left(\begin{array}{c}
n+m \\
n
\end{array}\right)+1\right)(\tilde{h}(F)+\tilde{h}(G))+\tilde{c} \max \{0,2 \mathfrak{g}-2+|S|\},
\end{aligned}
$$

which fits the assertion (1.2) with $\left(m_{1}, \ldots, m_{n}\right)=\mathbf{i}-\mathbf{i}_{0}$ and $\sum_{j=1}^{n}\left|m_{j}\right| \leq 2 m$.

We now consider when those $\mathbf{g}^{\mathbf{i}}$ with $\mathbf{i} \in \mathbb{Z}_{\geq 0}^{n}$ and $|\mathbf{i}| \leq m$ are linearly nondegenerate over $V_{F, G}(M r+1)$. By Theorem 26 combined with (6.31) and (6.32),

$$
\begin{aligned}
N_{S, \mathrm{gcd}}(F(\mathbf{g}), G(\mathbf{g})) & \leq\left(\frac{M^{\prime} m n}{M}+\left(\frac{w}{u}-1\right) m n\right) \max _{1 \leq i \leq n}\left\{h\left(g_{i}\right)\right\}+\tilde{c}_{1}(\tilde{h}(F)+\tilde{h}(G))+\tilde{c}_{2} \max \{0,2 \mathfrak{g}-2+|S|\} \\
& \leq \frac{\epsilon}{2} \max _{1 \leq i \leq n}\left\{h\left(g_{i}\right)\right\}+\tilde{c}_{1}(\tilde{h}(F)+\tilde{h}(G))+\tilde{c}_{2} \max \{0,2 \mathfrak{g}-2+|S|\},
\end{aligned}
$$


where $\tilde{c}_{1}$ and $\tilde{c}_{2}$ depend only on $\left(M_{m}, r\right)$, thus only on $\epsilon$. Hence, if

$$
\max _{1 \leq i \leq n}\left\{h\left(g_{i}\right)\right\} \geq \frac{4}{\epsilon}\left(\tilde{c}_{1}(\tilde{h}(F)+\tilde{h}(G))+\tilde{c}_{2} \max \{0,2 \mathfrak{g}-2+|S|\}\right),
$$

then

$$
N_{S, \operatorname{gcd}}(F(\mathbf{g}), G(\mathbf{g})) \leq \frac{3 \epsilon}{4} \max _{1 \leq i \leq n}\left\{h\left(g_{i}\right)\right\}
$$

We now estimate $h_{g c d}(F(\mathbf{g}), G(\mathbf{g}))$ using Theorem 27 with extra assumption that $F$ or $G$ does not vanish at the origin. We may assume that $F(0, \ldots, 0) \neq 0$. Since $m \geq 2 d$ and those $\mathbf{g}^{\mathbf{i}}$ with $\mathbf{i} \in \mathbb{Z}_{\geq 0}^{n}$ and $|\mathbf{i}| \leq m$ are linearly nondegenerate over $V_{F, G}(M r+1)$, it is clear that those $\mathbf{g}^{\mathbf{i}}$ with $\mathbf{i} \in \mathbb{Z}_{\geq 0}^{n}$ and $|\mathbf{i}| \leq d$ are linearly nondegenerate over $V_{F}(r)$. Then by Theorem 27 and (6.33), we have the the following

$$
\sum_{\mathbf{p} \in S} v_{\mathbf{p}}^{0}(F(\mathbf{g})) \leq \frac{\epsilon}{8} \max _{1 \leq i \leq n} h\left(g_{i}\right)+c_{1}^{\prime} h(F)+c_{2}^{\prime} \max \{0,2 \mathfrak{g}-2+|S|\},
$$

where $c_{1}^{\prime}:=\frac{w^{\prime}(N+1)}{u^{\prime}}(r+1)$ and $c_{2}^{\prime}:=\frac{w^{\prime}(N+1)\left(N w^{\prime}+w^{\prime}-1\right)}{2 u^{\prime}}$ with $N:=\left(\begin{array}{c}n+d \\ n\end{array}\right)-1$. Note that $c_{1}^{\prime}$ and $c_{2}^{\prime}$ depend only on $\left(w^{\prime}, u^{\prime}\right)$, thus only on $\epsilon$. By (6.39), we see that if both (6.37) and

$$
\max _{1 \leq i \leq n}\left\{h\left(g_{i}\right)\right\} \geq \frac{8}{\epsilon}\left(c_{1}^{\prime} h(F)+c_{2}^{\prime} \max \{0,2 \mathfrak{g}-2+|S|\}\right)
$$

hold, then

$$
\sum_{\mathbf{p} \in S} \min \left\{v_{\mathbf{p}}^{0}(F(\mathbf{g})), v_{\mathbf{p}}^{0}(G(\mathbf{g}))\right\} \leq \sum_{\mathbf{p} \in S} v_{\mathbf{p}}^{0}(F(\mathbf{g})) \leq \frac{\epsilon}{4} \max _{1 \leq i \leq n}\left\{h\left(g_{i}\right)\right\}
$$

and hence together with (6.38), we have

$$
h_{\mathrm{gcd}}(F(\mathbf{g}), G(\mathbf{g})) \leq \epsilon \max _{1 \leq i \leq n}\left\{h\left(g_{i}\right)\right\} .
$$

Proof of Theorem 10. Since $F, G \in \mathbf{k}\left[x_{1}, x_{2}\right]$, we have that $V_{F, G}(r)=V_{F}(r)=\mathbf{k}$ for each $r \in \mathbb{N}$. Given $\epsilon>0$, we first choose $m$ sufficiently large satisfying (6.31) with $n=2$. Suppose that those $g_{1}^{i_{1}} g_{2}^{i_{2}}$ with $\left(i_{1}, i_{2}\right) \in \mathbb{Z}_{\geq 0}^{2}$ and $i_{1}+i_{2} \leq m$ are linearly dependent over $\mathbf{k}$. Then there is a linear relation

$$
\sum_{\mathbf{j}=\left(j_{1}, j_{2}\right)} \alpha_{\mathbf{j}} g_{1}^{j_{1}} g_{2}^{j_{2}}=1
$$

where $\alpha_{\mathbf{j}} \in \mathbf{k}^{*}, \mathbf{j} \in \mathbb{Z}^{2} \backslash\{(0,0)\}$ and $\left|j_{1}\right|+\left|j_{2}\right| \leq 2 m$ for each appearing $\mathbf{j}=\left(j_{1}, j_{2}\right)$. We may also assume that no proper subsum of the left hand side of (6.40) vanishes. Consider the subgroup $J \subset \mathbb{Z}^{2}$ generated by those $\mathbf{j}$ appearing in (6.40). If $J$ has rank one, i.e., there exists $\left(m_{1}, m_{2}\right) \in \mathbb{Z}^{\mathbf{2}} \backslash\{(0,0)\}$ such that $\left(j_{1}, j_{2}\right)=\lambda_{\mathbf{j}}\left(m_{1}, m_{2}\right)$ with $\lambda_{\mathbf{j}} \in \mathbb{Z}$ for every $\mathbf{j}=\left(j_{1}, j_{2}\right)$ appearing in (6.40), then $\left|m_{1}\right|+\left|m_{2}\right| \leq 2 m$ and

$$
\sum_{\mathbf{j}} \alpha_{\mathbf{j}}\left(g_{1}^{m_{1}} g_{2}^{m_{2}}\right)^{\lambda_{\mathbf{j}}}=1
$$

which implies that $g_{1}^{m_{1}} g_{2}^{m_{2}} \in \mathbf{k}$. For the other cases, $J$ must have rank two, thus we can find $\left(j_{1}, j_{2}\right)$ and $\left(j_{1}^{\prime}, j_{2}^{\prime}\right)$ appearing in (6.40) such that $\mathbb{Q} \cdot\left(j_{1}, j_{2}\right) \neq \mathbb{Q} \cdot\left(j_{1}^{\prime}, j_{2}^{\prime}\right)$ (i.e. $\left(j_{1}, j_{2}\right)$ and $\left(j_{1}^{\prime}, j_{2}^{\prime}\right)$ are $\mathbb{Q}$-linearly 
independent), and

$$
\max \left\{h\left(g_{1}^{j_{1}} g_{2}^{j_{2}}\right), h\left(g_{1}^{j_{1}^{\prime}} g_{2}^{j_{2}^{\prime}}\right)\right\} \leq \frac{1}{2}\left(\left(\begin{array}{c}
m+2 \\
2
\end{array}\right)-1\right)\left(\left(\begin{array}{c}
m+2 \\
2
\end{array}\right)-2\right) \max \{0,2 \mathfrak{g}-2+|S|\}
$$

by using Theorem 14 Since $\mathbb{Q} \cdot\left(j_{1}, j_{2}\right) \neq \mathbb{Q} \cdot\left(j_{1}^{\prime}, j_{2}^{\prime}\right)$ and thus $j_{1} j_{2}^{\prime} \neq j_{1}^{\prime} j_{2}$, we note that $\left(j_{2}^{\prime} j_{1}-j_{2} j_{1}^{\prime}, 0\right)=$ $j_{2}^{\prime}\left(j_{1}, j_{2}\right)-j_{2}\left(j_{1}^{\prime}, j_{2}^{\prime}\right)$, thus by (6.41) we have

$$
\begin{aligned}
h\left(g_{1}\right) \leq h\left(g_{1}^{j_{2}^{\prime} j_{1}-j_{2} j_{1}^{\prime}}\right) & \leq\left|j_{2}^{\prime}\right| h\left(g_{1}^{j_{1}} g_{2}^{j_{2}}\right)+\left|j_{2}\right| h\left(g_{1}^{j_{1}^{\prime}} g_{2}^{j_{2}^{\prime}}\right) \\
& \leq 2 m\left(\left(\begin{array}{c}
m+2 \\
2
\end{array}\right)-1\right)\left(\left(\begin{array}{c}
m+2 \\
2
\end{array}\right)-2\right) \max \{0,2 \mathfrak{g}-2+|S|\} .
\end{aligned}
$$

With similar estimates for $h\left(g_{2}\right)$, this implies that $\max \left\{h\left(g_{1}\right), h\left(g_{2}\right)\right\} \leq c \max \{0,2 \mathfrak{g}-2+|S|\}$, where $c:=$ $2 m\left(\left(\begin{array}{c}m+2 \\ 2\end{array}\right)-1\right)\left(\left(\begin{array}{c}m+2 \\ 2\end{array}\right)-2\right)$ depends only on $\epsilon$.

In the case that those $g_{1}^{i_{1}} g_{2}^{i_{2}}$ with $\left(i_{1}, i_{2}\right) \in \mathbb{Z}_{\geq 0}^{2}$ and $i_{1}+i_{2} \leq m$ are linearly independent over $\mathbf{k}$, we shall conclude that $N_{S, \text { gcd }}\left(F\left(g_{1}, g_{2}\right), G\left(g_{1}, g_{2}\right)\right) \leq \epsilon \max \left\{h\left(g_{1}\right), h\left(g_{2}\right)\right\}$, and that $h_{\mathrm{gcd}}\left(F\left(g_{1}, g_{2}\right), G\left(g_{1}, g_{2}\right)\right) \leq \epsilon \max \left\{h\left(g_{1}\right), h\left(g_{2}\right)\right\}$ if we further assume that not both of $F$ and $G$ vanish at $(0,0)$. The corresponding part in the proof of Theorem 8 works, but actually an easier proof suffices. We omit the details.

Proof of Theorem 11, Let $S=S_{\mathbf{g}}:=\left\{\mathbf{p} \in C \mid v_{\mathbf{p}}\left(g_{i}\right) \neq 0\right.$ for $\left.1 \leq i \leq n\right\}$. Then, $g_{i} \in \mathcal{O}_{S}^{*}$ for each $1 \leq i \leq n$, and

$$
|S| \leq 2 \sum_{i=1}^{n} h\left(g_{i}\right) \leq 2 n \max _{1 \leq i \leq n}\left\{h\left(g_{i}\right)\right\} .
$$

Let $\epsilon>0$. Suppose that our assertion (i) (resp. (ii)) does not hold for some $\ell$. By Theorem 8 applied to $\left(g_{1}^{\ell}, \ldots, g_{n}^{\ell}\right) \in\left(\mathcal{O}_{S}^{*}\right)^{n}$, there exist an integer $m$, positive constants $c_{i}^{\prime}, 0 \leq i \leq 4$, all depending only on $\epsilon$, such that we have either

$$
\max _{1 \leq i \leq n} h\left(g_{i}^{\ell}\right) \leq c_{1}^{\prime}(\tilde{h}(F)+\tilde{h}(G))+c_{2}^{\prime} \max \{0,2 \mathfrak{g}-2+|S|\}
$$

or

$$
h\left(g_{1}^{\ell m_{1}} \cdots g_{n}^{\ell m_{n}}\right) \leq c_{3}^{\prime}(\tilde{h}(F)+\tilde{h}(G))+c_{4}^{\prime} \max \{0,2 \mathfrak{g}-2+|S|\}
$$

for some integers $m_{1}, \ldots, m_{n}$, not all zeros with $\sum\left|m_{i}\right| \leq 2 m$. If $g_{1}^{m_{1}} \cdots g_{n}^{m_{n}} \notin \mathbf{k}$, then we must have $\max _{1 \leq i \leq n} h\left(g_{i}\right) \geq 1$ and $h\left(g_{1}^{m_{1}} \cdots g_{n}^{m_{n}}\right) \geq 1$. Hence (6.42), (6.43) and (6.44) imply that

$$
\begin{aligned}
\ell & \leq\left(c_{1}^{\prime}+c_{3}^{\prime}\right)(\tilde{h}(F)+\tilde{h}(G))+\left(c_{2}^{\prime}+c_{4}^{\prime}\right) \max \{0,2 \mathfrak{g}-2+|S|\} \\
& \leq\left(c_{1}^{\prime}+c_{3}^{\prime}\right)(\tilde{h}(F)+\tilde{h}(G))+2\left(c_{2}^{\prime}+c_{4}^{\prime}\right)\left(\mathfrak{g}+n \max _{1 \leq i \leq n}\left\{h\left(g_{i}\right)\right\}\right) .
\end{aligned}
$$

This shows that our desired conclusion holds with $c_{1}:=c_{1}^{\prime}+c_{3}^{\prime}$ and $c_{2}:=2\left(c_{2}^{\prime}+c_{4}^{\prime}\right)$.

\section{REFERENCES}

[1] N. Ailon And Z. Rudnick, Torsion points on curves and common divisors of $a^{k}-1$ and $b^{k}-1$, Acta Arith. 113 (2004), no. $1,31-38$.

[2] T. T. H. An, H.-L. Huang And J. T.-Y. Wang, Generalized Büchi's problem for algebraic functions and meromorphic functions, Math. Z. 273 (2013), no. 1-2, 95-122. 
[3] W. D. Brownawell and D. W. Masser, Vanishing sums in function fields, Math. Proc. Cambridge Phil. Soc. 100 (1986), no. 3, 427-434

[4] P. Corvaja and U. Zannier, Diophantine equations with power sums and universal Hilbert sets, Indag. Math. (N.S.)9 (1998), no. 3, 317-332.

[5] P. Corvaja And U. Zannier, Some cases of Vojta's conjecture on integral points over function fields, J. Algebraic Geometry 17 (2008), no. 2, 295-333.

[6] N. Grieve And J. T.-Y. WAng, Greatest common divisors with moving targets and consequences for linear recurrence sequences, Transactions of A.M.S., to appear.

[7] A. Levin, Greatest common divisors and Vojta's conjecture for blowups of algebraic tori, Invent. Math. 215 (2019), no. $2,493-533$.

[8] A. Levin And J. T.-Y. WAng, Greatest common divisors of analytic functions and Nevanlinna theory on algebraic tori, J. Reine Angew. Math., to appear.

[9] A. Ostafe, On some extensions of the Ailon-Rudnick theorem, Monatsh. Math. 181 (2016), no. 2, 451-471.

[10] F. Pakovich and I. E. Shparlinski, Level curves of rational functions and unimodular points on rational curves, Proceedings of A.M.S. 148 (2020), no. 5, 1829-1833.

[11] H. PAsten, Powerful values of polynomials and a conjecture of Vojta, Journal of Number Theory 133 (2013), no. 9, 2964-2998.

[12] H. Pasten, J. T.-Y. WAng, Extensions of Büchi's higher powers problem to positive characteristic, Int. Math. Res. Not. (2015), no. 11, 3263-3297.

[13] J. T.-Y. WANG, An effective Roth's theorem for function fields, Rocky Mountain J. Math. 26 (1996), no. 3, $1225-1234$.

[14] J. T.-Y. WANG, An effective Schmidt's subspace theorem over function fields, Math. Z. 246 (2004), no. 4, 811-844.

[15] U. Zannier, A Proof of Pisot's d-th root conjecture, Ann. Math.(2) 151 (2000), no. 1, 375-383.

Institute of Mathematics, Academia Sinica, 6F, Astronomy-Mathematics Building, No. 1, Sec. 4, Roosevelt ROAD, TAIPEI 10617, TAIWAN

Email address: jiguo@math.sinica.edu.tw

Institute of Mathematics, Academia Sinica, 6F, Astronomy-Mathematics Building, No. 1, Sec. 4, Roosevelt RoAD, TAIPEI 10617, TAIWAN

Email address: csun@math.sinica.edu.tw

Institute of Mathematics, Academia Sinica, 6F, Astronomy-Mathematics Building, No. 1, Sec. 4, Roosevelt RoAD, TAIPEI 10617, TAIWAN

Email address: jwang@math.sinica.edu.tw 